\title{
1 The HIV-1 capsid core is an opportunistic nuclear import receptor
}

2

3 Guangai Xue ${ }^{1,6}$, Hyun Jae Yu¹, Shih Lin Goh², Anna T. Gres³, Mehmet Hakan Guney², Stefan G.

4 Sarafianos ${ }^{4,5}$, Jeremy Luban ${ }^{2}$, and Vineet N. KewalRamani ${ }^{1, *}$

5

$6 \quad{ }^{1}$ Basic Research Laboratory, National Cancer Institute, Frederick, MD, 21702, USA

$7 \quad{ }^{2}$ Program in Molecular Medicine, University of Massachusetts Medical School, Worcester, MA,

801605, USA

$9 \quad{ }^{3}$ Bond Life Sciences Center, Chemistry, University of Missouri, Columbia, MO, 65201, USA

$10{ }^{4}$ Bond Life Sciences Center, MMI, Biochemistry, University of Missouri, Columbia, MO, 65201,

11 USA

$12{ }^{5}$ Laboratory of Biochemical Pharmacology, Department of Pediatrics, Emory University School of

13 Medicine, Atlanta, GA, 30322, USA

$14{ }^{6}$ Present address: Department of Molecular Physiology and Biological Physics, University of

15 Virginia, Charlottesville, VA 22908, USA

16

17

18

19 email: vineet@mail.nih.gov

20 phone: $301-846-1249$ 
The movement of viruses and other large macromolecular cargo through nuclear pore complexes (NPCs) is poorly understood. The human immunodeficiency virus type 1 (HIV-1) provides an attractive model to interrogate this process due to the genetic and cell biological assays to score virus nuclear entry in living cells. Although initial studies of HIV-1 infection of nondividing cells focused on karyophilic virion proteins, subsequent work revealed the viral capsid (CA), the chief structural component of the pre-integration complex (PIC), to be a critical be altered through $\mathrm{CA}$ mutation ${ }^{2}$, which makes direct contact with nucleoporins $(\mathrm{Nups})^{3-5}$. Here we identify Nup35, Nup153, and POM121 to coordinately support HIV-1 nuclear entry. For Nup35 and POM121, this dependence was strongly dependent cyclophilin A (CypA) interaction with CA. Mutation of CA or removal of soluble host factors changed the interaction with the NPC. Collectively, these findings implicate the HIV-1 CA hexameric lattice that encapsulates the viral genome as a macromolecular nuclear transport receptor (NTR) that exploits soluble host factors to modulate NPC requirements during nuclear invasion. based on stoichiometry and distribution within the NPC (Extended Data Fig. 1a) ${ }^{6}$. Approximately onethird of Nups contain FG-dipeptides, motifs which are targets for NTR interaction for directional movement of cargo from the cytoplasm to the nucleus ${ }^{7}$. Genetic and biochemical studies have

41 implicated the FG-Nups Nup153 and Nup358 as contributing to HIV-1 nuclear entry ${ }^{5,8-11}$. These Nups

42 decorate opposite poles of the NPC with tentacles of Nup358 extending into the cytoplasm from the 43 outer nuclear membrane and Nup153 projecting a basket-like structure from the inner nuclear 44 membrane into the nucleoplasm. HIV-1 with mutations in CA that prevent interaction with soluble 
factors such as CPSF6 and CypA, exhibit a differential dependence on Nups during infection, including loss of reliance on both Nup153 and Nup358. CypA interacts with CA via the P90 residue exposed on loop in the N-terminal domain (NTD), and CPSF6 interacts via the N74 pocket within an NTD and Cterminal domain (CTD) interface present in hexameric CA in the virion core (Extended Data Fig. 1b and 1c). P90A and N74D mutations, respectively, impair CypA and CPSF6 interactions with CA. We considered the possibility that CPSF6 and CypA binding to HIV-1 in the cell cytoplasm affected subsequent interactions at the NPC and thus routes of nuclear entry.

To better understand the applicability of this interface exposure model, we performed a genetic screen with small-interfering RNAs (siRNAs) targeting 32 human Nups in HeLa cells to identify those that exhibited CA-dependence during infection with VSV-G pseudotyped HIV-1 vectors encoding red fluorescent protein (RFP). As a positive control to inhibit infection of HIV-1 with wild-type (WT) CA, TNPO3 was also depleted using siRNA ${ }^{8}$. Parallel infections were performed with HIV-1 CA mutant

(N74D and P90A) virus vectors and Moloney murine leukemia virus (MLV) vectors. Nup35, Nup153,

Nup358, and POM121 depletion were observed to inhibit WT HIV-1 infection but affected N74D and

P90A HIV-1 infection to a lesser degree (Fig. 1a). Nup35 and POM121 have not previously been studied as potential HIV-1 cofactors, and notably, their depletion had little effect on MLV infection (Extended Data Fig. 2). As has been previously reported ${ }^{2,11}$, Nup160 depletion affected WT and CA mutant HIV-1 infection, which is likely due to the critical role it plays in NPC formation. Nup58 knockdown enhanced infection of both WT and CA mutant HIV-1. MLV infection was not increased in Nup54, Nup58, Nup62, or Nup214 knockdown cells (Extended Data Fig. 2). Nup54, Nup58, Nup62, and Nup214 are notable due to their formation of an "FG-hydrogel" that provides a 

1 in a pattern similar to Nup153 and Nup358, we focused efforts on these two Nups. Initial studies were performed with Nup35 as it was less essential to N74D and P90A HIV-1 relative to POM121. Nup153 was selected over Nup358 as a control in subsequent experiments given significant cellular toxicity observed after Nup358 knockdowns. Nup153 was also of interest because HIV-1 CA engages it via an FG-motif.

To determine whether the effect of Nup35 on WT HIV-1 infection was direct, we sought to examine the stability of other Nups after Nup35 depletion with siRNA. Knockdown of Nup35 did not affect levels of other Nups present in the Nup93 subcomplex of which it is a member nor did it affect the levels of TNPO3, Nup153, or Nup358 (Extended Data Fig. 3). These data suggested that the indirect degradation of other Nups, including those known to interact with HIV-1, did not account for the infection block. In addition, Nup153 continues to associate with the nuclear membrane in Nup35 knockdown cells as does the Nup93 subcomplex constituent Nup155, in the same relative orientation to one another (Fig. 1b). Because N74D and P90A HIV-1 infection are unimpeded in Nup35-depleted cells, coupled with the microscopy data, these results indicate transient Nup35 loss did not grossly affect nuclear transport or NPC integrity.

We next tested three unique Nup35 siRNAs from the mixture used in the screen separately.

85 Consistent with the pooled siRNA results, two different siRNAs directed against Nup35, siRNA-2 and siRNA-3, reduced WT HIV-1 infection approximately 10-fold (Fig. 1c) correlating with Nup35 depletion, whereas N74D HIV-1 and P90A HIV-1 were resistant to Nup35 depletion.

To test whether the reductions in HIV-1 infection were due to off-target effects, we performed

89 a functional rescue experiment (Fig. 1d). Nup35 siRNA-3 targets 3'-UTR sequence. Thus HeLa cells 
91 that lacks the 3'-UTR and is therefore resistant to silencing by the siRNA. Western blot analysis

92 confirmed that siRNA knockdown reduced the endogenous level of Nup35 in both transduced cell

93 populations; however, ectopically expressed HA-Nup35 was unaffected by Nup35 siRNA treatment.

94 Consistent with prior results, cells depleted for endogenous Nup35 and transduced with the LPCX

95 empty vector were more than 10-fold less susceptible to HIV-1 infection. In contrast, Nup35 siRNA-

96 treated cells that expressed HA-Nup35 remained susceptible to WT HIV-1. N74D HIV-1 infection was

97 relatively consistent under the different Nup35 expression conditions. Notably, the small infection

98 decrease in MLV infection occurring after Nup35 knockdown was not offset in cells expressing

99 exogenous HA-Nup35 suggesting the effect on MLV could be indirect.

CRISPR/Cas9 gene-editing of nup35 in HeLa cells confirmed the siRNA findings. While 5

101 different guide RNAs (gRNAs) were tested, only one targeting the third exon of nup35 yielded cell

102 clones deficient of full-length Nup35. These clones uniformly expressed a truncated form of Nup35

103 that likely initiated from an in-frame methionine at coding residue position 62 of Nup35 within exon 3

104 based on the protein size and sequencing analysis (Fig. 1e and Extended Data Fig. 4a). The internally

105 initiated form of Nup35 may have permitted cell survival. These cells expressing the suspected $\mathrm{N}$ -

106 terminally truncated Nup35 had reduced susceptibility to HIV-1 infection but not N74D or P90A HIV-

1071 infection (Fig. 1e). Consistent with siRNA knockdown results, knockout of Nup35 did not affect

108 levels of other Nups present in the Nup93 subcomplex nor did it affect the levels of TNPO3, Nup153,

109 or Nup358 (Extended Data Fig. 4b). Knockout cell clones for Nup153 (Extended Data Fig. 4c) were

110 also obtained and were similarly less permissive for HIV-1 infection relative to N74D or P90A HIV-1

111 infection (Fig. 1f). Although we were unable to detect a truncated form of Nup153 in the knockout cells

112 by western blot analysis, it is possible that a shorter form permitting survival was not recognized by

113 our antibodies. 
Because N74D HIV-1 and P90A HIV-1 were insensitive to Nup35 depletion, we examined other viruses with CA mutations that influence interaction with CPSF6 or CypA for infection of Nup35 knockdown cells. HIV-1 with N57A, Q63A/Q67A, K70A, or T107A mutations in CA are reduced in sensitivity to CPSF6-mediated infection blocks and are similarly diminished in binding CPSF6 ${ }^{12}$. Indeed, relative to WT HIV-1, these viruses were less sensitive to TNPO3 depletion which enables CPSF6 inhibition of infection (Fig. 1g). While N57A, K70A, and N74D HIV-1 were similarly less sensitive to Nup35 depletion, Q63A/Q67A and T107A HIV-1 resembled WT HIV-1 in infection 121 indicating that CPSF6-interaction does not predict Nup35 dependence.

HIV-1, these viruses were impaired for infection of Nup35 and TNPO3 knockdown cells (Fig. 1g). We

125 similarly tested simian immunodeficiency virus from macaques $\left(\mathrm{SIV}_{\text {mac239}}\right)$, which replicates

126 independent of CypA interaction, and feline immunodeficiency virus (FIV) which does not interact

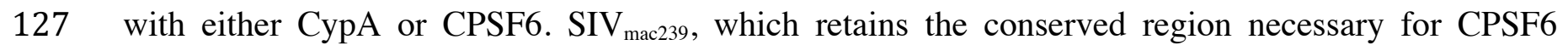
128 interaction, was potently impaired by TNPO3 knockdown but exhibited reduced dependence on Nup35

129 (Fig. 1g). FIV was unaffected by either Nup35 or TNPO3 knockdown. Taken together, these results 130 demonstrate that HIV-1 CA determines sensitivity to Nup35 depletion, and the ability to interact with 131 CypA correlated with dependence on Nup35.

133 CypA impairs HIV-1 infection in Nup35 knockdown cells. To directly test whether CypA was 134 required for the HIV-1 dependence on Nup35, we treated Nup35-knockdown cells with CsA to inhibit 135 the CypA-CA interaction (Fig. 2a). WT HIV-1 infectivity was rescued to the level of control cells by 136 CsA treatment in Nup35-knockdown cells. In contrast, CsA had minor effects on WT HIV-1 infection 
in either control cells or TNPO3-knockdown cells. N74D HIV-1 infection was previously demonstrated to be sensitive to CsA treatment ${ }^{15}$, and it remained sensitive in Nup35- and TNPO3-knockdown cells. As expected, the infectivity of HIV-1 with the CypA binding mutation P90A was not affected by CsA treatment in any cell type. These data demonstrate that CsA restores HIV-1 replication following Nup35

141 knockdown. The effect was specific to HIV-1, as $\mathrm{SIV}_{\text {mac239, }}$ FIV, and MLV were not affected by CsA 142 in Nup35-depleted cells (Fig. 2a). Taken together, these data indicate that the block to HIV-1 infection 143 in Nup35-depleted cells is dependent on the virus binding CypA. We sought to understand whether CypA affected WT HIV-1 dependence on Nup35 in the T cell line, MT4, which are highly permissive to transduction with shRNA-expressing lentiviral vectors. Cells

146 were transduced with GFP-encoding shRNA vectors targeting Nup35 and then challenged with HIV-1

147 in the absence and presence of CsA (Fig. 2b). While knockdown of Nup35 specifically diminished WT 148 HIV-1 infection in 2 of 3 shRNA lines, CsA treatment restored WT HIV-1 infectivity to levels observed 149 in shRNA control cells treated with the drug. These data underline the pivotal role of CypA in 150 determining the HIV-1 sensitivity to Nup35 depletion.

Given the interrelation between CypA binding and HIV-1 sensitivity to Nup35 depletion, we considered whether CsA-dependent viruses, A92E and G94D HIV-1, would remain sensitive to Nup35

153 depletion after CsA treatment. The CypA-dependent infection blocks that A92E and G94D HIV-1

154 exhibit in HeLa cells have been previously characterized as occurring at nuclear entry ${ }^{16}$. In cells 155 depleted of TNPO3, CsA treatment fails to fully restore the replication of these viruses indicating a 156 qualitatively different infection block (data not shown). In comparison, A92E and G94D HIV-1 were 157 also additionally sensitive to loss of Nup35 in cells (Fig. 2c). However in the presence of CsA, A92E 158 and G94D HIV-1 infectivity in Nup35 knockdown cells rises to the level of the mutant viruses in control 159 cells treated with CsA. Collectively, these data suggest the mechanisms underlying HIV-1 infection 
160 inhibition in Nup35 knockdown cells may be similar to the infection blocks encountered by A92E and

161 G94D HIV-1 in normal cells in that CypA drives these viruses toward a nuclear entry pathway that

162 they are unable to exploit.

164 HIV-1 infection in POM121 and Nup153 knockdown cells is affected by CypA. The degree by

165 which CypA controlled HIV-1 sensitivity to Nup35 depletion led us to investigate whether HIV-1

166 interactions with POM121, which emerged from the same screen, and Nup153 were CypA-dependent.

167 Cells were either individually treated with siRNAs targeting Nup35, POM121, Nup153, and CypA, or

168 doubly treated with siRNAs targeting Nup35, POM121, and Nup153 in combination with siRNA

169 targeting CypA. Notably, depletion of CypA not only restored HIV-1 infection in Nup35 knockdown

170 cells but similarly restored infection in POM121 knockdown cells and partly restored infection in

171 Nup153 knockdown cells (Fig. 2d). A92E HIV-1 was also largely insensitive to the different Nup

172 knockdowns in cells where CypA was also depleted. By contrast, MLV infection was largely unaffected

173 in cells with single Nup knockdowns or Nup knockdowns in combination with CypA knockdowns

174 (Extended Data Fig. 5).

175

176 Nup35 knockdown impairs HIV-1 nuclear entry. To gain insight into the specific stage of HIV-1

177 infection at which Nup35 and CsA exert their effects, we performed time course experiments on

178 Nup35-knockdown HeLa cells that were treated with CsA at various times after exposure to virus. Up

179 to 12 hours post infection of Nup35-depleted cells, WT HIV-1 infectivity could be rescued by CsA

180 treatment; however, CsA completely lost rescue ability at 24 hours post-infection (Fig. 3a). Similarly,

181 CsA treatment also rescued the infectivity of CsA-dependent HIV-1 mutant A92E 12 hours post 182 infection, but could not rescue infectivity at 24 hours post infection. P90A HIV-1 and MLV infection 
183 were not affected by CsA treatment at any time point, which is consistent with the inability of P90A

184 HIV-1 and MLV to bind CypA.

185 Given the early reversibility of the infection block and the alteration of the nuclear pore complex

186 by the various Nup knockdowns, we evaluated HIV-1 reverse transcription in cells depleted of Nup35

187 in the absence or presence of CsA (Extended Data Fig. 6 and Fig. 3b). We observed no difference in

188 the accumulation of early or late reverse transcription products in control cells vs. Nup35-knockdown

189 cells (Fig. 3b). Notably, both WT and A92E HIV-1 exhibited significantly reduced levels of 2-LTR

190 circle junction forms of viral DNA (vDNA) unless treated with CsA. Although 2-LTR circular vDNA

191 represents an abortive, noninfectious path, they form in the nucleus in the presence of DNA Ligase IV

192 and thus can be used to assess viral nuclear entry ${ }^{17}$. These data indicate that the Nup35 knockdown does

193 not impair reverse transcription but prevents HIV-1 nuclear entry in the presence of CypA.

194 We next tested whether CypA also regulated HIV-1 nuclear entry in cells depleted of POM121

195 or Nup153 using an assay based on CPSF6 distribution in HIV-1 infected cells. These cells were

196 depleted of Nup35, POM121, or Nup153 in the absence of presence of CsA. As before, WT and A92E

197 HIV-1 were sensitive to depletion of these Nups, and this infection reduction could be eliminated

198 through CsA treatment (Fig. 3c). By contrast, MLV infection was not affected by CsA in Nup35,

199 POM121, or Nup153 depleted cells (data not shown). Because CA interacts with CPSF6, soon after

200 infection, it can induce CPSF6 precipitates, especially in the nucleus where CPSF6 is abundant (Fig.

201 3d). In Nup35-knockdown cells, WT HIV-1 infection no longer causes CPSF6 precipitation in the 202 nucleus (Fig. 3e). Conversely, CsA treatment recovered CPSF6 aggregation following WT HIV-1

203 infection. As with the reverse-transcription staging data, these results indicate that restoration of HIV-

2041 infection by CsA is linked to nuclear entry. Similar results were obtained with A92E HIV-1. Notably,

205 this virus did not induce nuclear aggregation of CPSF6 in control cells unless in the presence of CsA. 
As expected, CsA treatment did not alter CPSF6 distribution in P90A HIV-1 infected control cells,

such that CPSF6 aggregates were observed in Nup35-knockdown cells in the absence of CsA. The phenotypes of WT HIV-1, P90A HIV-1, and A92E HIV-1 were consistent in cells depleted of either POM121 (Fig. 3f) or Nup153 (Fig. 3g). These results summarized in Fig. 3h indicate a pivotal role of CypA in regulating HIV-1 nuclear entry in Nup35, Nup153, and POM121 knockdown cells.

regulatory switch. Given the pivotal role that soluble factor binding appeared to exert on transport of HIV-1 through the NPC, we considered whether differences in the subcellular localization of CPSF6 and CypA could predict their impact on this process. Although present throughout the cell, CypA is enriched in cytoplasmic compartments, especially in proximity to the nuclear membrane in both HeLa cells and T cell lines (Fig. 4a and Extended Data Fig. 7a-c). This distribution appears relatively stable and is not grossly affected by Nup35-depletion (Extended Data Fig. 7d). CPSF6, consistent with its role in pre-mRNA processing, is predominantly nuclear and distributed in a pattern quite distinct from CypA and is also unaffected by Nup35 depletion (Extended Data Fig. 7e). POM121 knockdown cells. HIV-1 infection in these cells can be fully restored after CsA treatment. In

224 comparison, P90A HIV-1 does not interact with CypA, and the infectivity of this virus is slightly 225 diminished in POM121 and Nup153 knockdown cells (Fig. 1a, Fig. 3c, and Fig. 4b). Whereas CsA 226 treatment, predictably, does little to affect P90A infection in the POM121 or Nup153 knockdown cells, 227 CPSF6 depletion potently enhances infection when in conjunction with either knockdown (Fig. 4b). 
Although WT HIV-1 infection in Nup35 knockdown cells was elevated after CPSF6 knockdown, infection in Nup153 knockdown cells remained impaired even after CPSF6 knockdown, suggesting distinct contributions of both Nups to HIV-1 nuclear entry. These observations were confirmed and extended. While the effect of TNPO3 knockdown on HIV-1 infection could be significantly ameliorated by CPSF6 depletion, and the effect of Nup35 knockdown on HIV-1 infection could be partially offset by CPSF6 depletion, the reduction in HIV-1 infection by Nup153 knockdown potently impaired WT but not N74D HIV-1 infection.

We next asked whether CypA was also a key regulator of Nup155 use. Nup155 is a non-FG infection than WT HIV-12. WT HIV-1 infection is diminished in cells depleted of Nup153 but retains when CypA is depleted in Nup153 and Nup155 knockdown cells. WT HIV-1 infection is increased in

241 the Nup153 knockdown cells as previously noted, and it is decreased in the Nup155 knockdown cells 242 (Fig. 4d). The infectivity of WT HIV-1 in the CypA plus Nup155 double knockdown cells is 243 comparable to N74D and P90A HIV-1 infection of the Nup155 cells. In the absence of CypA, it

244 becomes more dependent on Nup155. These data suggest the use of a secondary nuclear pathway for 245 HIV-1 when insertion into the Nup153 pathway is not possible.

247 linked to soluble host factor binding, it remains possible that these proteins also make direct contact

248 with HIV-1 despite their position within the NPC. We thus sought to examine whether fragments of 249 Nup35 or POM121 could interfere with HIV-1 in a CA-dependent manner. This approach has been 250 leveraged in mapping CA-interaction domains in CPSF6 and Nup153, specifically through fusion to 
251 the N-terminal RING, B-box 2, and coiled-coil (RBCC) domains of rhesus TRIM5 alpha, substituting

252 the B30.2 (SPRY) domain with a region of interest ${ }^{10,18}$. Attempts to make rhTRIM5-Nup35 fusions

253 that were detectable in cell lines were unsuccessful. By contrast, rhTRIM5 fusions to the N-terminus

254 and C-terminus of POM121 were stable (Fig. 4e). Due to the potency of rhTRIM5-mediated restriction

255 and the sensitivity of WT, N74D, and P90A HIV-1 to POM121 depletion (Fig. 1a), we first tested

256 whether HIV-1 and MLV were differentially susceptible to infection in cells expressing the TRIM5-

257 POM121 fusion proteins (Fig. 4e). Whereas MLV displayed no apparent sensitivity to the different

258 TRIM5-POM121 proteins, HIV-1 (LAI Gag) was specifically inhibited by a TRIM5 fusion to the FG-

259 rich, C-terminal domain of POM121. Moreover, testing with HIV-1/MLV Gag chimeric viruses

260 revealed that HIV-1 CA was required for sensitivity to this protein. We extended this analysis to the

261 CA-mutant HIV-1 (NL4-3 Gag) isolates (Extended Data Fig. 8). Although HIV-1 CA governs

262 susceptibility to TRIM5-POM121-Cterm, the N74D and P90Amutations were insufficient to escape

263 restriction by the fusion protein. 


\section{Discussion}

Here we demonstrate the dependence of HIV-1 on Nup35 and POM121 for nuclear entry. As has been observed for Nup153 and Nup358, HIV-1 CA determines sensitivity to loss of Nup35 or

POM121. Extending prior work, soluble CA-interacting factors also regulate the necessity for the nucleoporins. Unlike smaller cargo that interacts with cellular NTRs to achieve nuclear entry, we

271 propose that the HIV-1 core, comprised of multimeric CA in association with the viral nucleic acid and enzymatic proteins, directly functions as a macromolecular NTR and negotiates multiple nucleoporin interactions to achieve transfer through the NPC, through a pathway determined by soluble host factor binding in the cytoplasm. Like cellular NTRs, CA selectively interacts with FG dipeptides ${ }^{10,12}$. In contrast to cellular NTRs, HIV-1 can enter the nucleus of metabolically inert cells without being dependent on Ran-GTP regulation. Given that the host cell cytoplasm contains sensors to detect and interfere with microbial pathogens, slipping into the nucleus quickly is advantageous to the virus.

281 factors present in the cytoplasm known to interact with CA include CPSF6, CypA, and MxB. MxB is 
chromatin. Prior studies have indicated that it is detrimental to the virus to engage these factors out of order. Enrichment of CPSF6 in the cell cytoplasm blocks HIV-1 nuclear entry presumably by preventing Nup interactions with the virus ${ }^{2,20}$.

The role of CypA in HIV-1 infection has been a long-standing puzzle. It was one of the first mapped to exert its effect on early replication steps prior to nuclear entry ${ }^{22,23}$, the precise mechanism has been unclear. Studies with Nup153 and Nup358 suggested that CypA could affect subsequent interactions with these Nups ${ }^{5,24}$, but to a limited extent. It is possible that the essential role that these

Nups play in the transport of all cargo limits analysis after they are depleted from cells. By contrast,

297 the effect of CypA on HIV-1 dependence of Nup35 or POM121 was dramatic. While HIV-1 was 298 blocked for infection in Nup35 or POM121 knockdown cells, after treatment with CsA, HIV-1 enters 299 the nucleus and infection is restored to levels of control cells. CypA-binding thus directed HIV-1 to a 300 nonproductive nuclear import pathway in the knockdown cells, possibly one that starts with Nup358

301 interaction. Thus, CypA might not only block premature CPSF6 interaction before docking at the pore, 302 its association with HIV-1 might facilitate a transfer of the core to Nup358, initiating a specific path of 303 passage through the NPC.

At this point, it is unknown whether Nup35 and POM121 make direct contact with HIV-1. Nup35 is a member of the Nup93 subcomplex which encircles the central channel Nup62 subcomplex comprised entirely of FG-Nups. Nup35 itself has three FG dipeptides, but it is unknown if these are accessible to cargo. Given the demonstrated interaction of FG dipeptides in CPSF6 and Nup153 with the N74 pocket of HIV-1 CA, it is tempting to speculate that this interface of HIV-1 negotiates a 309 multitude of interactions by the core during passage through the NPC. To a large extent, Nup35 and 310 POM121 knockdowns phenocopied Nup153 knockdowns with regards to CA-dependent effects on 
311 HIV-1 infection. Similar to Nup153 and CPSF6, TRIM5 fusions to the FG-rich portion of POM121

312 inhibit HIV-1 infection. Recently, another group has also shown that a fragment of highly related

313 POM121C containing numerous FG-motifs was sufficient to impair HIV-1 infection ${ }^{25}$. While they did

314 not investigate whether POM121/POM121C could serve as a co-factor through knockdown/knockout

315 experiments, they argue that overexpression of truncated POM121C binds to a subunit of a cellular

316 NTR, Karyopherin subunit beta-1 (KPNB1), which possibly underlies a block to HIV-1 infection.

317 KPNB1 forms a complex with Importin-7 to mediate transport of cargo to the nucleus ${ }^{26}$. KPNB1

318 interaction with POM121C is not unexpected given that NTRs interact with FG-Nups. More recent

319 studies have not supported a role for Importin-7 in HIV-1 infection ${ }^{27}$. Consistent with this finding, we

320 also did not observe removal of either KPNB1 or Importin-7 to effect HIV-1 infection (Extended Data

321 Fig. 9). Instead POM121 interaction with CA appears to support HIV-1 infection.

322 Notably, POM121 is known to interact with Sun1 ${ }^{28}$. Sun1 and Sun2 combine to form a linker

323 of nucleoskeleton and cytoskeleton (LINC) complexes which span the nuclear envelope ${ }^{29}$. Knockdown

324 or knockout of Sun2 reduces HIV-1 infection in human primary CD4+ T cells ${ }^{30}$ and THP-1 cells ${ }^{31}$,

325 respectively. The CypA-dependence of Sun2 is unsettled. One study has shown that Sun2 promotes

326 CypA-dependent steps of HIV-1 replication in bone marrow-derived dendritic cells from sun2-/-

327 mice ${ }^{30}$, but other two studies have shown that there is no correlation between Sun2 and CypA in human

328 primary CD4+ T cells ${ }^{32}$ and THP-1 cells ${ }^{31}$. These differences could be due to cell context. Sun 1 and

329 Sun2 form an inner nuclear membrane (INM) complex proximal to NPCs. It will be important to

330 determine the contribution of INM proteins with nucleoporins in influencing HIV-1 nuclear entry

331 pathway. How Sun2 interacts with HIV-1 is a subject of investigation.

332 Elucidating the precise choreography of HIV-1 interactions at the nuclear membrane and during

333 passage through the NPC is critical to understanding the successive contributions of each of these 
334 factors. As illustrated in Figure 4c, the Nup35 and Nup153 blocks to HIV-1 infection were additive in

335 double-knockdown cells. One interpretation of these data is that the blocks are distinct and Nup35 loss

336 does not entirely prevent Nup153 use by HIV-1. It could be argued that the removal of both factors was

337 incomplete, so the effect of the double knockdown was increased potency in inhibiting the same

338 pathway. However, the genetics of both blocks are distinct. While there may be an overlapping need

339 for function by both Nup35 and Nup153 for optimal HIV-1 nuclear entry and infection, CPSF6,

340 potentially interacting with the virus within the NPC, creates a more stringent requirement on Nup35

341 (Fig. 4c) indicating functional differences between successive steps during nuclear import.

342 Indeed our prior work pointed to the existence of distinct routes of nuclear entry available to

343 HIV - $1^{2}$, which split along an axis of WT vs N74D HIV-1 CA exhibiting greater dependence on Nup153

344 vs Nup155, respectively. We find here that this switch in pathway use is governed in part by CypA.

345 N74D HIV-1 exhibits great sensitivity to the loss of CypA with an infection block in the cytoplasm ${ }^{15}$.

346 When Nup155 loss is coupled with CypA depletion in experiments presented here, a greater than 50-

347 fold decrease in N74D HIV-1 infectivity is observed. Moreover, WT HIV-1 also becomes more

348 dependent on the Nup155-import pathway in CypA-depleted cells. For WT HIV-1, a loss of CypA

349 binding diminishes access to the Nup153-import pathway. For N74D HIV-1, the N74D mutation of CA

350 precludes Nup153 utilization.

A competition between CypA and CPSF6 for binding to HIV-1 CA likely exists in the cell

352 cytoplasm given the overlap of binding sites. CypA is one of the most abundant proteins in the cell.

353 With the elevated concentration of CypA proximal the nuclear membrane, HIV-1 may have evolved to

354 bind it to prevent premature interaction with CPSF6 (Fig. 4f). CPSF6 aggregates are in fact observed

355 in the cytoplasm of cells infected with P90A HIV-1 (Fig. 3f-g). The affinity of full-length CPSF6

356 interaction with CA hexamers is currently unknown, although a peptide of the CA-binding site of 
CPSF6 has an estimated affinity $\left(\mathrm{K}_{\mathrm{D}}\right)$ of 50 micromolar ${ }^{4}$. CypA binding to the CA lattice at an affinity $\left(\mathrm{K}_{\mathrm{D}}\right)$ of approximately 12 micromolar ${ }^{33}$. Thus it is unlikely that CPSF6 will exhibit preferential binding to cores in areas of the cell where CypA is at higher concentration.

While both HIV-1 and SIVsm/mac/mne viruses retain CPSF6 binding interfaces, HIV-1

361 distinctly acquired a CypA interaction site in proximity to the CPSF6 binding interface. This potentially

362 reflects differences in the subcellular localization of CPSF6 in human vs some nonhuman primate cells.

363 If human cells have elevated CPSF6 levels outside the nucleus, this could impair HIV-1 interaction

364 with the NPC. As we observe here (e.g. Fig. 2a,b) and has been noted in past studies ${ }^{13,14}$, CsA treatment

of some human cell targets results in diminished WT HIV-1 infection. Despite the potential danger of

HIV-1 infection (Fig. 4b,c). How to reconcile both CypA and CPSF6 effects on HIV-1 infection when interaction with Nup358 at the cytoplasmic face of the NPC, previously hypothesized by Schaller et al

CPSF6 and probably a subset of FG-Nups to subsequently interact with CA hexamers. This would unidentified, surfaces on CA to negotiate potential nucleoporin interactions. themes. Removal of Nup62 subcomplex members (Nup54, Nup58, and Nup62), which are FG-Nups

377 that form a meshwork in the central channel of the pore restricting the flow of cargo larger than $5 \mathrm{~nm}$ 378 in diameter, generally elevated both WT and N74D HIV-1 infection. While loss of Nup54 and Nup58 379 elevated infection of WT HIV-1, N74D HIV-1, and P90A HIV-1, removal of Nup62 had a larger 
positive effect on WT HIV-1 infection. Knockdown of Nup214, a FG-Nup that also is thought to regulate the flow of cargo, also had a greater positive effect on WT HIV-1 infection. Collectively, WT HIV-1 infection appears to be more broadly regulated by FG-Nups. Although we did not directly investigate the role of CypA in facilitating Nup62 and Nup214 interaction, P90A HIV-1 infection was 2-3-fold lower than WT HIV-1 infection under knockdown conditions. The ability of CypA/CPSF6 to regulate HIV-1 interactions with the NPC underscores the vulnerability of the virus at this crucial replication step to $\mathrm{MxB}$ which also targets HIV-1 CA ${ }^{34-36}$. Both N74D ${ }^{34,35}$ and $\mathrm{P} 90 \mathrm{~A}^{34} \mathrm{HIV}-1$ show increased resistance to MxB antiviral function. Similarly, other CypA and CPSF6 CA-binding mutants G89V and N57S, respectively, also exhibit MxB resistance ${ }^{35}$. $\mathrm{MxB}$ restriction has been associated with CypA function ${ }^{36}$, and it is proposed that the block occurs at the nuclear entry ${ }^{34,35}$. The potential interplay of MxB with CypA or CPSF6, and whether it dysregulates the temporal or spatial sequence of their interactions with CA, has not been examined at this point. CA is intrinsic to critical replication steps in the cytoplasm, at the nuclear membrane, and within the nucleus. Many of these actions are likely dependent on retention of a portion of the core, especially

394 given the N74 interface is accessed by factors when CA is in a hexameric configuration. The coordination of different successive interactions among a limited subset of interfaces helps explain why that this choreography is profoundly sensitive to disruption by small molecule inhibitors targeting CA, such as PF-74 and BI-237,38.

Taken together, this study supports a model of HIV-1 CA co-opting CypA as a key factor in 399 preventing premature interactions in the cytoplasm by CPSF6 and presumably newly synthesized, 400 soluble nucleoporins until the virus reaches the nuclear membrane. Subsequently, the CA lattice 401 functions as a NTR which binds to different FG motifs present in FG-Nups or CPSF6 in the nuclear 402 channel enabling HIV-1 transport into the nucleus. The transposon Tf1 is similarly hypothesized to use 
403 an assembled Gag particle as a multimeric NTR, particularly in interacting with the FG-rich yeast

404 Nup124p during nuclear entry ${ }^{39}$. With regards to animal cell biology, as our and previous studies

405 indicate, HIV-1 provides an attractive model to interrogate the mechanism of nuclear entry by large 406 macromolecular entities.

407

408

409

410 


\section{METHODS}

413 Plasmids. pNL4-3-Luc-E-R+ (HIV-1 vpr-positive, env-deleted, encoding firefly luciferase in place of 414 nef) or pHIV-RFP (HIV-1 vpr-negative, env-deleted, encoding RFP in place of nef) were used in 415 transfections with pL-VSV-G to generate VSV-G-pseudotyped HIV-1 vectors, and the MLV-based 416 retroviral vector pMX-RFP was used to generate Moloney-based virus in co-transfections with pJK3, 417 pL-VSV-G, and pCMV-Tat. Vectors for FIV ${ }^{40}$ and $\operatorname{SIV}_{\text {MAC239 }}{ }^{41}$ have been described previously. cDNA encoding human Nup35 was amplified from MGC Human Nup35 sequence-verified cDNA (Accession number BC047029, GE Healthcare, Dharmacon), using primers that add an amino 420 terminal HA tag, and inserted into pLPCX-MCS between the EcoRI and NotI sites. All coding 421 sequences were confirmed by DNA sequencing.

Cells and culture conditions. HEK293T and HeLa cell lines were maintained in DMEM supplemented with 10\% FBS. H9, Jurkat, and MT4 cells were grown in RPMI 1640 plus 10\% FBS. GHOST cells were maintained in DMEM supplemented with 10\% FBS, $500 \mathrm{ug} / \mathrm{ml} \mathrm{G418,} 100 \mathrm{ug} / \mathrm{ml}$ hygromycin, and $1 \mathrm{ug} / \mathrm{ml}$ puromycin.

To generate stable Nup35-expressing cell lines, HeLa cells were transduced with either wildtype or mutant HA-tagged Nup35, a pLPCX-HA-Nup35 (cds) vector containing only the amino acid

429 coding sequence of the human Nup35 cDNA, which excludes the 3'-UTR, and the cells were selected 430 with $1 \mu \mathrm{g} / \mathrm{ml}$ puromycin (Millipore).

432 siRNA screen. To identify Nups requirements for HIV-1 infection, 32 human Nups were 433 systematically depleted using two different commercially available siRNA pools (Dharmacon; Sigma). 
434 siRNAs were transfected into the HeLa cells at a $50 \mathrm{nM}$ final concentration using RNAiMAX

435 (ThermoFisher Scientific), according to the manufacturer's instructions. After 2 days, the cells were

436 re-seeded into 24-well plates and infected with VSV-G-pseudotyped RFP reporter viruses at an MOI

437 of 0.5 in the presence of $5 \mathrm{ug} / \mathrm{ml}$ polybrene. After an additional $48 \mathrm{~h}$ incubation, cells were trypsinized

438 and the percentage of RFP-positive cells was scored by flow cytometry (FACSCalibur, BD

439 Biosciences). As a positive control, siRNA SMARTpool against TNPO3 (Dharmacon) was present on

440 each plate. To exclude false positives (e.g., due to toxicity), WT HIV-1, N74D HIV-1, and P90A HIV-

4411 infections were performed in parallel to Moloney murine leukemia virus (MLV) infection on the

442 Nup-depleted cells. The first round of the screens was performed using siRNA from Sigma. Those

443 genes that showed a phenotype in the first round were confirmed by Dharmacon siRNA. Therefore,

444 two-thirds of the genes were screened using both Dharmacon on-target siRNA SMARTpool and Sigma

445 MISSION esiRNA. The screening was repeated in at least three independent experiments.

447 Viral production. To produce HIV-1 particles, pNL4-3-Luc-E-R+ or HIV-RFP (wild-type and capsid 448 mutants: N57A, Q63/67A, K70A, N74D, P90A, A92E, G94D, and T107A) was co-transfected with a 449 VSV-G expression vector at a ratio of 3:1 using Hilymax (Dojindo Molecular Technologies). The 450 medium was replaced after overnight incubation and viral supernatants were collected at $48 \mathrm{~h}$ post-

451 transfection. Viral supernatants were filtered and their infectivity was determined by using HeLa or

452 GHOST target cells.

453 MLV stocks were obtained by co-transfection of pLPCX, pJK3, pL-VSV-G, and pCMV-Tat at 454 a ratio of 4:2:1:0.3, respectively, with Hilymax. The medium was replaced one day after overnight 455 incubation and viruses were harvested at $48 \mathrm{~h}$ after transfection, passed through a $0.45-\mu \mathrm{m}$ filter, and 456 used directly to transduce target cells. 
458 Viral infection. For single cycle infectivity assays, HeLa cells were plated in 24 -well plates at $5 \times 10^{4}$

459 cells per well, and infected with single or serial-dilutions of VSV-G-pseudotyped HIV-1, SIVmac mas $_{239}$

$460 \mathrm{FIV}$, or MLV in the presence of $5 \mathrm{ug} / \mathrm{ml}$ polybrene. In some experiments, $2.5 \mu \mathrm{M}$ cyclosporine A

461 (Bedford laboratories) was added to the culture media at the time of infection. At $48 \mathrm{~h}$ post-infection,

462 cells were washed, and the infection was analyzed by examining the percentage of RFP or GFP

463 expressing cells using flow cytometry (FACSCalibur, BD Biosciences), or by measuring firefly

464 luciferase activity (Promega).

465 For microscopy experiments, $2.5 \times 10^{4} \mathrm{HeLa}$ cells were grown overnight on glass slides in a 24-

466 well plate. The next day, cells were infected with WT, P90A, or A92E pNL4-3-Luc-E-R+ at an MOI

467 of 50 . At $12 \mathrm{~h}$ post-infection, cells were washed three times with PBS, fixed, permeabilized, and stained

468 with antibodies.

For analysis of HIV-1 reverse transcription products, HeLa cells were seeded at $2 \times 10^{5}$ cells per

470 well in 6-well plates and infected with VSV-G-pseudotyped wild-type HIV-1 or capsid mutant virus

471 (MOI of 1) in the presence or absence of $2.5 \mu \mathrm{M}$ cyclosporine A. For the negative control, a reverse

472 transcription inhibitor (EFV $150 \mathrm{nM}$ ) was added at the time of infection. The phenotype infection assay

473 was performed in parallel in 24-well plates.

474 RNA interference. Three ON-TARGETplus siRNAs directed against human Nup35 were purchased

475 from Dharmacon: siRNA 1, 5'-CUGCUGGUUCCUUCGGUUA-3'; $\quad$ siRNA 2, 5'-

476 AGAUAAAAGUGGCGCUCCA-3'; siRNA 3, 5'-AGUUAUUUCUACC GACACA-3'. HeLa cells

477 were plated at $1.5 \times 10^{5}$ cells per well in 6-well plates and transfected the next day with a final

478 concentration of $40 \mathrm{nM}$ siRNA targeting Nup35 or non-targeting control siRNA (siCONTROL non-

479 targeting siRNA, Dharmacon), using RNAiMAX (ThermoFisher Scientific) according to the 
480 manufacturer's instructions. To generate stable Nup35 knockdown cells, a lentiviral vector and the

481 following GIPZ Lentiviral (GE Healthcare, Dharmacon) shRNAs against hNup35 were used in this

482 study:

483 GIPZ Lentiviral Human Nup35 shRNA:

484 V3LHS_364366, TGTCTGTCAGAAATAACCT

485 V3LHS_380787, TATGAGCTGGTACAACTGG

486 V3LHS_380788, TGGTTCAGATCCTAACGCG

487 Lentiviral vector stocks were produced by co-transfection of 293T cells with packaging plasmid $\Delta \mathrm{R} 8.2$,

488 MISSION shRNA or GIPZ Lentiviral shRNA, and pL-VSV-G at a ratio of 1:1:0.5, the culture medium

489 was replaced at $12 \mathrm{~h}$, and viral supernatants were collected and filtered at $48 \mathrm{~h}$. HeLa cells were

490 transduced with the filtered supernatant and selected in $2 \mathrm{ug} / \mathrm{ml}$ puromycin.

492 CRISPR-Cas9 knockout.

493 To generate a CRISPR-Cas9 single guide RNA (sgRNA) expression vector, oligonucleotides were 494 annealed and inserted into the pX330 (Addgene plasmid \#42230). The following sequences targeting 495 coding regions at the 5' end of the gene were used: 5'-GAAGGGCCACTAATTGATCG-3' (Nup35, 496 exon3) and 5'-GGACGCGGCGTTGCCACCAG-3' (Nup153, exon1). Non-targeting sgRNA was used 497 as control, 5'-CGCTTCCGCGGCCCGTTCAA-3'. HeLa knockout cells were generated by transiently 498 transfecting pX330 into the cells. Single clones were screened by western blotting and phenotype assay 499 and the candidate clones were verified by sequencing of genomic DNA using the following primers: 
taAAGCTTGTCCCATACCTGATGCTGTTGT-3' (Nup153 target exon1).

RNAi-resistant mutant generation and phenotype rescue. To generate RNAi-resistant variants of genes, the exogenous ORF transcript lacking the 3'-UTR sequence targeted by the siRNA was inserted into LPCX vector. HeLa cells were then transduced with the filtered supernatant and the cells were selected with $1 \mu \mathrm{g} / \mathrm{ml}$ puromycin (Millipore). For the rescue assay, puromycin-selected cells were plated at $1.5 \times 10^{5}$ cells per well in 6-well plates and transfected the next day with siRNA (40 nM) against human Nup35 3'-UTR region or control siRNA. Two days post-transfection, cells were re-seeded and

511 infected with the HIV-1 or MLV and analyzed by FACS 2 days post-infection.

513 Quantification of HIV-1 reverse transcription products by real-time PCR. HeLa cells were

514 transfected with siRNAs as described above. Two days later, cells were seeded at $2 \times 10^{5}$ cells per well

515 in 6-well plates and infected with VSV-G-pseudotyped HIV-1, or capsid mutant virus P90A HIV-1, or

516 A92E HIV-1. The virus was pretreated with $20 \mathrm{U} \mathrm{ml}^{-1} \mathrm{RNase}-$ free DNase I (Roche) for $1 \mathrm{~h}$ at $37^{\circ} \mathrm{C}$. At

$5172 \mathrm{~h}$ post-infection, the cells were washed once with PBS and fresh complete medium was added, cells

518 were then collected at 3, 6, 12 and $24 \mathrm{~h}$ after infection. Total DNA was extracted using the QIAamp

519 DNA Blood Mini Kit (Qiagen) and used for real-time PCR to specifically quantify HIV early reverse

520 transcription (RT) products, late RT products, and 2-LTR circle forms. The primer-probe sets and

521 conditions were used as previously described ${ }^{42}$. To normalize the amount of DNA in each PCR assay,

522 the following primer set was used to quantify the copy number of the cellular gene actin: forward, 5'-

523 TCACCCACACTGTGCCCATCTA CGA $\quad 3^{\prime}$ and $\quad$ reverse $5^{\prime}-$

524 CAGCGGAACCGCTCATTGCCAATGG -3'. qPCR assays were performed using either Platinum 
525 qPCR SuperMix-UDG (Invitrogen) for detecting HIV-1 reverse transcription or iQ SYBR Green

526 Supermix (Bio-Rad) for detecting actin.

528 Western blotting. Whole-cell extracts were prepared by lysing cells in RIPA buffer (Sigma-Aldrich), 529 equivalent protein content boiled in SDS sample buffer, resolved by Criterion Tris-HCl Precast Gels 530 (Bio-Rad), and blotted onto PVDF Blotting Membranes (GE Healthcare). Membranes were probed 531 with primary antibodies specific for human cyclophilin A (Abcam), CPSF6 (Novusbio), Importin 7 532 (Novusbio), KPNB1 (Novusbio), Nup35/53 (Abcam, Bethyl, GeneTex, or Novusbio), Nup93 (Abcam),

533 Nup153 (Abcam), Nup155 (Abcam), Nup188 (Novusbio), Nup205 (Novusbio), Nup358 (Abcam), 534 POM121 (GeneTex), TNPO3 (MyBioSource), HA (Sigma), and tubulin (Sigma) followed by 535 secondary HRP-conjugated anti-mouse (GE Healthcare) or anti-rabbit (GE Healthcare) antibodies and 536 detected using a Chemidoc XRS+ system (Bio-Rad).

Immunofluorescence. Cells were fixed with $4 \%$ paraformaldehyde (Boston Bioproducts) at room 539 temperature (RT) for $10 \mathrm{~min}$, permeabilized with $0.2 \%$ Triton X-100 in PBS for 10 min, and then 540 blocked with $3 \%$ BSA for $30 \mathrm{~min}$. Cells were incubated with primary antibodies at RT for $1 \mathrm{~h}$, followed 541 by Alexa-Fluor-conjugated secondary antibodies and Hoechst 33342 (Thermo Fisher Scientific) at RT

542 for 30 min. Coverslips were mounted on glass slides with ProLong Gold antifade solutions (Molecular

543 Probes). Images were taken in Z-stacks using the Deltavison deconvolve microscope (GE Healthcare)

544 and deconvolved to remove out-of-focus light using the Softworks software (GE Healthcare). Primary

545 and secondary antibodies used for immunofluorescence were mouse anti-Cyclophilin A (Abcam), 546 rabbit anti-CPSF6 (Novusbio), mouse anti-Nup153 (Abcam), Rabbit anti-Nup155 (Abcam), mouse 547 anti-Nuclear Pore Complex (MAb 414, BioLegend), rabbit anti-Lamin B1 (Abcam), goat anti-rabbit 
548 Alexa 488 (Molecular Probes), goat anti-mouse 488 (Molecular Probes), goat anti-mouse 546

549 (Molecular Probes), and goat anti-rabbit 555 (Molecular Probes).

\section{FIGURE LEGENDS}

553 Figure 1. HIV-1 dependence on Nup35. a, CA-dependent use of nucleoporins by HIV-1. HeLa cells

554 were transfected with a control siRNA or target siRNA (smart pool) for $48 \mathrm{~h}$, then infected with m.o.i 555 of 0.5 of VSV-G-pseudotyped wild-type (WT) virus or virus harboring various CA mutants. After 48

556 h, infected RFP-positive cells were counted by FACS. Nups targeted by siRNAs are grouped by NPC

557 subcomplexes. TNPO3 siRNA-treated cells are included in all the screens as a positive control. WT

558 HIV-1 infection decreases of greater than 3-fold after knockdown of nucleoporins are encircled. Percent

559 relative infection is mean \pm s.d., from three independent experiments. MLV infection was also

560 measured at $48 \mathrm{~h}$ in parallel samples to monitor cell toxicity and specificity of HIV infection (Extended

561 Data Fig. 2). b, Distribution of Nup153 and Nup155 after Nup35 knockdown. Localization of Nup153

562 (red) and Nup155 (green) in HeLa cells by deconvolution microscopy after immunostaining. Lamin B1

563 is blue. A Z-section image is presented. c, different siRNAs targeting Nup35 impair HIV-1 infection.

564 HeLa cells were transfected with a control siRNA or three different siRNAs against Nup35 for 48 h,

565 then infected with VSV-G-pseudotyped WT HIV-1 vectors or virus harboring various CA mutants or

566 MLV. After $48 \mathrm{~h}$, infected cells expressing fluorescent markers from viral vectors were enumerated by

567 FACS. Error bars show standard deviations of duplicates, representative of three independent

568 experiments. Western blot analysis confirmed Nup35 depletion by three different siRNAs. d, HeLa

569 cells stably expressing human HA-Nup35 lacking 3'-UTR or transduced with control vector (LPCX-

570 HA), were transfected with non-target (NT) siRNA or Nup35 siRNA targeting 3'-UTR region for 48 
571 h, then infected with VSV-G-pseudotyped virus. Infected RFP-positive cells were counted by FACS.

572 Error bars show standard deviations of duplicates, representative of three independent experiments.

573 Western blot confirmed Nup35 restoration. e, f, HIV-1 CA determines Nup35 (e) or Nup153 (f)

574 dependence in HeLa knockout cell clones. Nup35 or Nup153 knockout HeLa cell clones were infected

575 by HIV-1 or MLV. Infected RFP-positive cells were counted by FACS $48 \mathrm{~h}$ after infection. Error bars

576 show standard deviations of duplicates, representative of three independent experiments. Western blots

577 show the knockout efficiency of Nup35 (e) or Nup153 (f). Empty, cell lines that only transiently co-

578 transfected pX330 with puromycin-expression vector, plasmid; NT, non-targeting control lines lacking

579 a gene-specific gRNA. g, HeLa cells were transfected with control siRNA or siRNAs against Nup35

580 or TNPO3 for $48 \mathrm{~h}$, then infected with VSV-G-pseudotyped pNL4-3-Luc-E-R+ or virus harboring

581 various CA mutants, or various retroviruses as labeled. Firefly luciferase activity and GFP/RFP-

582 positive cells were monitored at $48 \mathrm{~h}$. Error bars show standard deviations of duplicates, representative

583 of two independent experiments. Western blotting confirmed knockdown efficiency.

585 Figure 2. HIV-1 dependence on Nup35, Nup153, and POM121 is regulated by CypA. a, HIV-1,

586 SIV, FIV, and MLV infection in the absence or presence of CsA in Nup35-depleted cells. HeLa cells

587 were transfected with control siRNA or siRNAs against Nup35 for $48 \mathrm{~h}$, then infected with VSV-G-

588 pseudotyped viruses in the presence or absence of $2.5 \mu \mathrm{M} \mathrm{CsA}$. After $48 \mathrm{~h}$, infected GFP/RFP-positive

589 cells were counted by FACS. Error bars show standard deviations of duplicates, representative of two

590 independent experiments. Western blotting confirmed knockdown efficiency. b, CsA restores HIV-1

591 infection in Nup35-depleted MT4 cells. MT4 cells were transduced with a control shRNA or three

592 different shRNAs against Nup35 then infected with VSV-G-pseudotyped WT or virus harboring

593 various CA mutants. Firefly luciferase activity was measured at $48 \mathrm{~h}$. Error bars show standard 
594 deviations of duplicates, representative of two independent experiments. Western blot analysis

595 confirmed Nup35 depletion by three different shRNAs. c, CsA restores A92E and G94D HIV-1

596 infectivity in Nup35 knockdown cells. HeLa cells were transfected with control siRNA or siRNA

597 against Nup35 for $48 \mathrm{~h}$, then infected with increasing amounts of VSV-G-pseudotyped viruses in the

598 presence or absence of $2.5 \mu \mathrm{M} \mathrm{CsA}$, and firefly luciferase infectivity was measured at $48 \mathrm{~h}$. Error bars

599 show standard deviations of duplicates, representative of three independent experiments. d, CypA

600 depletion by siRNA restores HIV-1 infectivity in Nup35 and POM121 knockdown HeLa cells. HeLa

601 cells were either single-knocked down (Nup35, POM121, Nup153, or CypA) or double-knocked down

602 (CypA in combination with Nup35, POM121 or Nup153) for 48 h. The cells were infected with VSV-

603 G-pseudotyped pNL4-3-Luc-E-R+ or CA mutants (P90A or A92E) and firefly luciferase infectivity

604 was measured at $48 \mathrm{~h}$. MLV infection was also measured at $48 \mathrm{~h}$ in parallel samples to monitor cell

605 toxicity and specificity of HIV-1 infection (Extended Data Fig. 5). Error bars show standard deviations

606 of duplicates, representative of three independent experiments.

608 Figure 3. Nup35 knockdown impairs HIV-1 nuclear entry. a, CsA can restore HIV-1 infectivity in

609 Nup35 knockdown cells $12 \mathrm{~h}$ after virus challenge. HeLa cells were transfected with control siRNA or

610 siRNA against Nup35 for $48 \mathrm{~h}$, then infected with VSV-G-pseudotyped viruses. CsA was added at

611 every $2 \mathrm{~h}$ until $12 \mathrm{~h}$ post-infection and at $24 \mathrm{~h}$ post-infection. Firefly luciferase activity was measured

612 at 48 h. Error bars show standard deviations of duplicates, representative of two independent

613 experiments. b, HIV-1 reverse transcription is blocked in Nup35 knockdown cells but 2-LTR circle

614 forms are not observed. HeLa cells were transfected with control or Nup35 siRNA for $48 \mathrm{~h}$, then

615 infected with VSV-G-pseudotyped, DNase-treated NL4-3-Luc-E-R+ or CA mutants (P90A or A92E)

616 at an MOI of 1 . The Cell DNA was extracted at 3, 6, 12, and $24 \mathrm{~h}$ after infection and used to detect 
617 early (RU5) RT, late (2nd strand) RT, and 2-LTR circles (right). Error bars show standard deviations

618 of duplicates, representative of two independent experiments. The amount of DNA in each PCR assay

619 was normalized by actin. HIV-1 infection was also measured at $48 \mathrm{~h}$ in parallel samples to monitor

620 HIV-1 infectivity (Extended Data Fig. 6). c, CsA restores HIV-1 infection in Nup35, Nup153, and

621 POM121 knockdown HeLa cells. HeLa cells were transfected with control siRNA or siRNAs against

622 Nup35, POM121, or Nup153 for 48 h, then infected with infected with VSV-G-pseudotyped NL4-3-

623 Luc-E-R+ or CA mutants (P90A or A92E) viruses in the presence or absence of $2.5 \mu \mathrm{M}$ CsA. After 48

624 h, firefly luciferase infectivity was measured. Error bars show standard deviations of duplicates,

625 representative of three independent experiments. Western blotting confirmed knockdown efficiency. -

626 , no CsA; +, 2.5 uM CsA. d-g, CPSF6 distribution of Nup35, POM121, and Nup153 knockdown cells

627 in the presence or absence of $2.5 \mu \mathrm{M}$ CsA. Cells were infected with VSV-G-pseudotyped NL4-3-Luc-

628 E-R+ or CA mutants (P90A or A92E) at an MOI of 50. After $12 \mathrm{~h}$, cells were fixed and stained with

629 antibodies against CPSF6 and NPC. h, The number of cells with or without CPSF6 aggregates were

630 counted from $(\mathbf{d}-\mathbf{g})$.

Figure. 4. Soluble factors regulate nucleoporin dependence. a, CypA enrichment at the nuclear

633 periphery. Localization of CPSF6 (green) and CypA (red) in HeLa cells in a Z-section image from

634 deconvolution microscopy of immunostained HeLa cells. b, Partial restoration of HIV-1 infection in

635 Nup35 and POM121 knockdown cells after CPSF6 depletion. HeLa cells were either single-knocked

636 down (Nup35, POM121, Nup153, or CPSF6) or double-knocked down (CPSF6 with Nup35, POM121

637 or Nup153) for $48 \mathrm{~h}$. The cells were infected with VSV-G-pseudotyped NL4-3-Luc-E-R+ or CA 638 mutants (N74D or P90A) viruses in the presence or absence of $2.5 \mu \mathrm{M}$ CsA, and firefly luciferase 639 infectivity and percentage of RFP-positive cells (MLV infection) were measured at $48 \mathrm{~h}$. Error bars 
640 show standard deviations of duplicates, representative of three independent experiments. Western

641 blotting confirmed knockdown efficiency. c, Distinct contributions of Nup35 and Nup153 to HIV-1

642 infection. HeLa cells were either single-knocked down (CPSF6, TNPO3, Nup35, or Nup153) or double-

643 knocked down (CPSF6 with TNPO3, Nup35, or Nup153; Nup35 with Nup153) for 48 h. The cells were

644 infected with VSV-G-pseudotyped HIV-RFP or CA mutant (N74D) viruses, and percentage of RFP-

645 positive cells were measured at $48 \mathrm{~h}$. Error bars show standard deviations of duplicates, representative

646 of three independent experiments. Western blotting confirmed knockdown efficiency. d, CypA

647 determines HIV-1 dependency on Nup153 versus Nup155. HeLa cells were either single-knocked 648 down (CypA, Nup153, or Nup155) or double-knocked down (CypA with Nup153 or Nup155) for 48

649 h. The cells were infected with VSV-G-pseudotyped pNL4-3-Luc-E-R+ or CA mutants (N74D or 650 P90A) viruses, and firefly luciferase infectivity and percentage of RFP-positive cells (MLV infection)

651 were measured at 48 h. Error bars show standard deviations of duplicates, representative of three 652 independent experiments. Western blotting confirmed knockdown efficiency. e, TRIM5-POM121

653 inhibition of HIV-1 infection is CA-dependent. Top left, schematic representation of the rhesus TRIM5

654 RBCC (RING, B-box2, and coiled coil) domains (1-299) with the POM121 N-terminal fragments (1655 438) or C-terminal fragments (439-984). Top right, western blotting analysis of lysates from HeLa cells 656 expressing TRIM fusion proteins. Bottom left, schematic representation of the chimeric HIV/MLV 657 virus. Bottom right, HeLa cells stably transduced with HA-tagged rhTrim5a/POM121 fusion constructs 658 were infected with VSV-G-pseudotyped chimeric HIV/MLV virus, and firefly luciferase infectivity 659 was measured at $48 \mathrm{~h}$. Error bars show standard deviations of duplicates, representative of three 660 independent experiments. Western blot analysis of HA-tagged rhTrim5a/POM121 fusion and control 661 constructs in stable HeLa cells (Extended Data Fig. 8). f, CypA determines the HIV-1 nuclear import 662 pathway. Premature CPSF6 interaction with HIV-1 impairs CA interaction with FG-Nups at the NPC. 
663 CypA use by HIV-1, however, prevents CPSF6 access to the N74 pocket in CA until the virus docks

664 at the NPC. Subsequent CPSF6 binding may enhance release from the NPC. We propose that the HIV-

6651 core, comprised of multimeric CA in association with the viral nucleic acid and enzymatic proteins,

666 directly functions as a NTR and exploits successive FG interactions to achieve transfer through the

667 NPC but these interactions are regulated in time and space by CypA and CPSF6.

668

669 Acknowledgements

670 We thank Szu-Wei Huang and KyeongEun Lee for intellectual contributions and scientific

671 discussions. We thank Eric Freed, Henry Levin, and Owen Pornillos for scientific suggestions, and

672 Eric Freed for MT4 cells. We thank Joseph Meyer from Scientific Publications, Graphics and

673 Media, Frederick National Laboratory for assistance with figure graphics. Dr. Xue was an NIH

674 Intramural AIDS Research Fellowship and NCI Sallie Rosen Kaplan Award recipient for women

675 scientists. This research was supported by the Intramural Research Program of the NIH,

676 Frederick National Lab, Center for Cancer Research. This work was also supported by NIH grants

677 5R01AI111809, 5DP1DA034990, and 1R01AI117839, to J.L. S.G.S. acknowledges support by NIH

678 grants P50 GM103368 and R01 AI120860. The content of this publication does not necessarily

679 reflect the views or policies of the Department of Health and Human Services, nor does mention

680 of trade names, commercial products, or organizations imply endorsement by the U.S.

681 Government.

682

683 Extended Data Legends

684 
Extended Data Figure 1: Depiction of the nuclear pore complex and HIV-1 CA hexamers. a,

Major human NPC subcomplexes. FG-Nups hypothesized to interact with nuclear pore cargo based on

687 yeast or metazoan studies are emboldened. Image adapted from Ori et $a l^{6} . \mathbf{b}$ and c, Overlap of CypA

688 and CPSF6 binding regions in the HIV-1 capsid. Surface representation of the structure of two adjacent

689 hexamers from the cryo-EM model of capsid (CA) tubes (PDB ID: 3J34; yellow CA $\mathrm{NTDS}_{\text {and orange }}$

690 CA $_{\text {CTDS }}$ ). (b) Binding site of a CPSF6 peptide (residues 313-327; magenta) based on crystal structures

691 PDB ID: 4U0A and 4WYM. (c) Binding site of CypA based on cryo-EM model PDB ID: 5FJB; blue.

692 The structures suggest that CypA bound to capsid may affect access of the full size CPSF6 to its binding

693 site.

Extended Data Figure 2: Effect of nucleoporin knockdown on MLV infection. HeLa cells were

transfected with a control siRNA or target siRNA (smart pool) for $48 \mathrm{~h}$, then infected with m.o.i of 0.5

of VSV-G-pseudotyped MLV. After 48 h, infected RFP-positive cells were counted by FACS. This

analysis was performed in parallel the experiments shown in Fig. 1a. Percent relative infection is mean

$699 \pm$ s.d., from three independent experiments. MLV, murine leukaemia virus.

Extended Data Figure 3: Nup35 knockdown does not affect levels of other nucleoporins. Nup35

702 knockdown does not affect levels of other proteins in the Nup93 subcomplex. Western blot analysis

703 with anti-Nup35 (a) or antibodies against Nup93 subcomplex proteins (b) using lysates from Nup35-

704 knockdown HeLa cells. c, Nup153 or Nup358 levels are unchanged in Nup35 knockdown cells.

705 Western blot analysis showing reactivity to anti-Nup35 or antibodies against TNPO3, Nup153, and 
Extended Data Figure 4: Generation of knockout HeLa cells. a, Genotyping of nup35 knockout clonal HeLa cell line. The protospacer adjacent motif (PAM) region is shown in orange, and the CRISPR-Cas9 nuclease target sequence in blue. Gene editing events are indicated in red at the bottom

711 of the wild-type sequence. b, Expression of nucleoporins in Nup35 knockout cells. Western blot analysis of Nup93 subcomplex proteins as well as TNPO3, Nup153, and Nup358 from Nup35 knockout in orange, and the CRISPR-Cas9 nuclease target sequence in blue. Gene editing events are indicated in red at the bottom of the wild-type sequence.

721 analysis was performed in parallel to the experiments shown in Fig. 2d. Error bars show standard

722 deviations of duplicates, representative of three independent experiments. Western blotting confirmed 723 knockdown efficiency.

Extended Data Figure 6: Nup35 knockdown impairs HIV-1 nuclear entry. HeLa cells were amounts of VSV-G-pseudotyped viruses in the presence or absence of $2.5 \mu \mathrm{M}$ CsA. Firefly luciferase activity and percentage of RFP-positive cells (MLV infection) were measured at $48 \mathrm{~h}$. Western blotting

729 confirmed knockdown efficiency. This analysis was performed in parallel the experiments shown in $730 \quad$ Fig. 3b. 
Extended Data Figure 7: Subcellular distribution of soluble factors. a-c, Distribution of CPSF6 and CypA in MT4 (a), Jurkat (b), and H9 (c) cells. Localization of CPSF6 (green), CypA (red), and lamin B1 (blue) in a Z-section of immunostained cells imaged by deconvolution microscopy. d, Nup35

735 depletion does not alter CypA subcellular localization. Localization of CypA (green) and lamin B1

736 (red) in a Z-section of immunostained HeLa cells imaged by deconvolution microscopy. e, Nup35

737 depletion does not alter CPSF6 subcellular localization. Localization of CPSF6 (green) and lamin B1 738 (red) in a Z-section of immunostained HeLa cells imaged by deconvolution microscopy.

741 infection. HeLa cells stably transduced with HA-tagged rhTrim5a/POM121 fusion constructs were 742 infected with VSV-G-pseudotyped HIV-RFP or CA mutants (N74D or P90A) viruses, and percentage 743 of RFP-positive cells was measured at $48 \mathrm{~h}$.

745 Extended Data Figure 9: Efficient HIV-1 infection in cells depleted of KPNB1 or Importin-7.

746 HeLa cells were transfected with control siRNA or siRNAs against TNPO3, POM121, KPNB1, or

747 Importin 7 for 48 h, then infected with VSV-G-pseudotyped HIV-RFP or MX-RFP. After 48 h, RFP748 positive cells were enumerated by FACS. Error bars show standard deviations of duplicates, 749 representative of two independent experiments. Western blotting confirmed knockdown efficiency. 


\section{REFERENCES}

752

7531 Yamashita, M., Perez, O., Hope, T. J. \& Emerman, M. Evidence for direct involvement of

754

755

756

757

758 the capsid protein in HIV infection of nondividing cells. PLoS Pathog 3, 1502-1510,

759

760

761 doi:10.1371/journal.ppat.0030156 (2007).

2 Lee, K. et al. Flexible use of nuclear import pathways by HIV-1. Cell Host Microbe 7, 221233, doi:10.1016/j.chom.2010.02.007 (2010).

3 Matreyek, K. A. \& Engelman, A. Viral and cellular requirements for the nuclear entry of retroviral preintegration nucleoprotein complexes. Viruses 5, 2483-2511, doi:10.3390/v5102483 (2013).

4 Price, A. J. et al. Host cofactors and pharmacologic ligands share an essential interface in HIV-1 capsid that is lost upon disassembly. PLoS Pathog 10, e1004459, doi:10.1371/journal.ppat.1004459 (2014). Schaller, T. et al. HIV-1 capsid-cyclophilin interactions determine nuclear import pathway, integration targeting and replication efficiency. PLoS Pathog 7, e1002439, doi:10.1371/journal.ppat.1002439 (2011). stoichiometry of molecular machines. Mol Syst Biol 9, 648, doi:10.1038/msb.2013.4 (2013).

7 Terry, L. J., Shows, E. B. \& Wente, S. R. Crossing the nuclear envelope: hierarchical regulation of nucleocytoplasmic transport. Science 318, 1412-1416,

8 Brass, A. L. et al. Identification of host proteins required for HIV infection through a functional genomic screen. Science 319, 921-926, doi:10.1126/science.1152725 (2008). Konig, R. et al. Global analysis of host-pathogen interactions that regulate early-stage HIV-1 replication. Cell 135, 49-60, doi:10.1016/j.cell.2008.07.032 (2008). Matreyek, K. A., Yucel, S. S., Li, X. \& Engelman, A. Nucleoporin NUP153 phenylalanineglycine motifs engage a common binding pocket within the HIV-1 capsid protein to mediate lentiviral infectivity. PLoS Pathog 9, e1003693, doi:10.1371/journal.ppat.1003693 (2013). 1 infection. PLoS Pathog 10, e1003969, doi:10.1371/journal.ppat.1003969 (2014). Price, A. J. et al. CPSF6 defines a conserved capsid interface that modulates HIV-1 replication. PLoS Pathog 8, e1002896, doi:10.1371/journal.ppat.1002896 (2012).

13 Sokolskaja, E., Sayah, D. M. \& Luban, J. Target cell cyclophilin A modulates human immunodeficiency virus type 1 infectivity. J Virol 78, 12800-12808, doi:10.1128/JVI.78.23.12800-12808.2004 (2004).

14 Hatziioannou, T., Perez-Caballero, D., Cowan, S. \& Bieniasz, P. D. Cyclophilin interactions with incoming human immunodeficiency virus type 1 capsids with opposing effects on infectivity in human cells. J Virol 79, 176-183, doi:10.1128/JVI.79.1.176-183.2005 (2005).

15 Ambrose, Z. et al. Human immunodeficiency virus type 1 capsid mutation N74D alters cyclophilin A dependence and impairs macrophage infection. J Virol 86, 4708-4714, doi:10.1128/JVI.05887-11 (2012). 
De Iaco, A. \& Luban, J. Cyclophilin A promotes HIV-1 reverse transcription but its effect on transduction correlates best with its effect on nuclear entry of viral cDNA. Retrovirology 11, 11, doi:10.1186/1742-4690-11-11 (2014).

17 Li, L. et al. Role of the non-homologous DNA end joining pathway in the early steps of retroviral infection. EMBO J 20, 3272-3281, doi:10.1093/emboj/20.12.3272 (2001).

18 Lee, K. et al. HIV-1 capsid-targeting domain of cleavage and polyadenylation specificity factor 6. J Virol 86, 3851-3860, doi:10.1128/JVI.06607-11 (2012). nuclear targeting signal and is localized in the heterochromatin region beneath the nuclear envelope. J Biol Chem 271, 23478-23486 (1996). De Iaco, A. et al. TNPO3 protects HIV-1 replication from CPSF6-mediated capsid stabilization in the host cell cytoplasm. Retrovirology 10, 20, doi:10.1186/1742-469010-20 (2013).

21 Luban, J., Bossolt, K. L., Franke, E. K., Kalpana, G. V. \& Goff, S. P. Human immunodeficiency virus type 1 Gag protein binds to cyclophilins A and B. Cell 73, 1067-1078 (1993).

22 Braaten, D., Franke, E. K. \& Luban, J. Cyclophilin A is required for an early step in the life cycle of human immunodeficiency virus type 1 before the initiation of reverse transcription. J Virol 70, 3551-3560 (1996).

814

24 Matreyek, K. A. \& Engelman, A. The requirement for nucleoporin NUP153 during human

23 Song, C. \& Aiken, C. Analysis of human cell heterokaryons demonstrates that target cell restriction of cyclosporine-resistant human immunodeficiency virus type 1 mutants is genetically dominant. J Virol 81, 11946-11956, doi:10.1128/JVI.00620-07 (2007). immunodeficiency virus type 1 infection is determined by the viral capsid. J Virol $\mathbf{8 5}$, 7818-7827, doi:10.1128/JVI.00325-11 (2011).

823 P0M121C inhibits HIV-1 replication. PLoS One 12, e0182434, doi:10.1371/journal.pone.0182434 (2017). Jakel, S. et al. The importin beta/importin 7 heterodimer is a functional nuclear import receptor for histone H1. EMBO J 18, 2411-2423, doi:10.1093/emboj/18.9.2411 (1999). Zielske, S. P. \& Stevenson, M. Importin 7 may be dispensable for human immunodeficiency virus type 1 and simian immunodeficiency virus infection of primary macrophages. J Virol 79, 11541-11546, doi:10.1128/JVI.79.17.11541-11546.2005 (2005).

28 Talamas, J. A. \& Hetzer, M. W. POM121 and Sun1 play a role in early steps of interphase NPC assembly. J Cell Biol 194, 27-37, doi:10.1083/jcb.201012154 (2011).

29 Sosa, B. A., Rothballer, A., Kutay, U. \& Schwartz, T. U. LINC complexes form by binding of three KASH peptides to domain interfaces of trimeric SUN proteins. Cell 149, 1035-1047, doi:10.1016/j.cell.2012.03.046 (2012).

30 Lahaye, X. et al. Nuclear Envelope Protein SUN2 Promotes Cyclophilin-A-Dependent Steps of HIV Replication. Cell Rep, doi:10.1016/j.celrep.2016.03.074 (2016).

31 Schaller, T. et al. Effects of Inner Nuclear Membrane Proteins SUN1/UNC-84A and SUN2/UNC-84B on the Early Steps of HIV-1 Infection. J Virol 91, doi:10.1128/JVI.0046317 (2017). Proliferation and Alters Sensitivity to HIV-1 Infection Independently of Cyclophilin A.J Virol 91, doi:10.1128/JVI.02303-16 (2017). 
33 Goldstone, D. C. et al. Structural studies of postentry restriction factors reveal antiparallel dimers that enable avid binding to the HIV-1 capsid lattice. Proc Natl Acad Sci U S A 111, 9609-9614, doi:10.1073/pnas.1402448111 (2014).

34 Goujon, C. et al. Human MX2 is an interferon-induced post-entry inhibitor of HIV-1 infection. Nature 502, 559-562, doi:10.1038/nature12542 (2013).

35 Kane, M. et al. MX2 is an interferon-induced inhibitor of HIV-1 infection. Nature 502, 563-566, doi:10.1038/nature12653 (2013).

36 Liu, Z. et al. The interferon-inducible MxB protein inhibits HIV-1 infection. Cell Host Microbe 14, 398-410, doi:10.1016/j.chom.2013.08.015 (2013).

37 Blair, W. S. et al. HIV capsid is a tractable target for small molecule therapeutic intervention. PLoS Pathog 6, e1001220, doi:10.1371/journal.ppat.1001220 (2010).

38 Lamorte, L. et al. Discovery of novel small-molecule HIV-1 replication inhibitors that stabilize capsid complexes. Antimicrob Agents Chemother 57, 4622-4631, doi:10.1128/AAC.00985-13 (2013).

39 Dang, V. D. \& Levin, H. L. Nuclear import of the retrotransposon Tf1 is governed by a nuclear localization signal that possesses a unique requirement for the FXFG nuclear pore factor Nup124p. Mol Cell Biol 20, 7798-7812 (2000).

40 Kemler, I., Barraza, R. \& Poeschla, E. M. Mapping the encapsidation determinants of feline immunodeficiency virus. J Virol 76, 11889-11903 (2002). Kim, S. S. et al. Generation of replication-defective helper-free vectors based on simian immunodeficiency virus. Virology 282, 154-167, doi:10.1006/viro.2000.0808 (2001). Julias, J. G., Ferris, A. L., Boyer, P. L. \& Hughes, S. H. Replication of phenotypically mixed human immunodeficiency virus type 1 virions containing catalytically active and catalytically inactive reverse transcriptase. J Virol 75, 6537-6546, doi:10.1128/JVI.75.14.6537-6546.2001 (2001). 
bioRxiv preprint doi: https://doi.org/10.1101/2021.12.02.470925; this version posted December 2, 2021. The copyright holder for this preprint (which was not certified by peer review) is the author/funder, who has granted bioRxiv a license to display the preprint in perpetuity. It is made Figure 1 available under aCC-BY 4.0 International license.
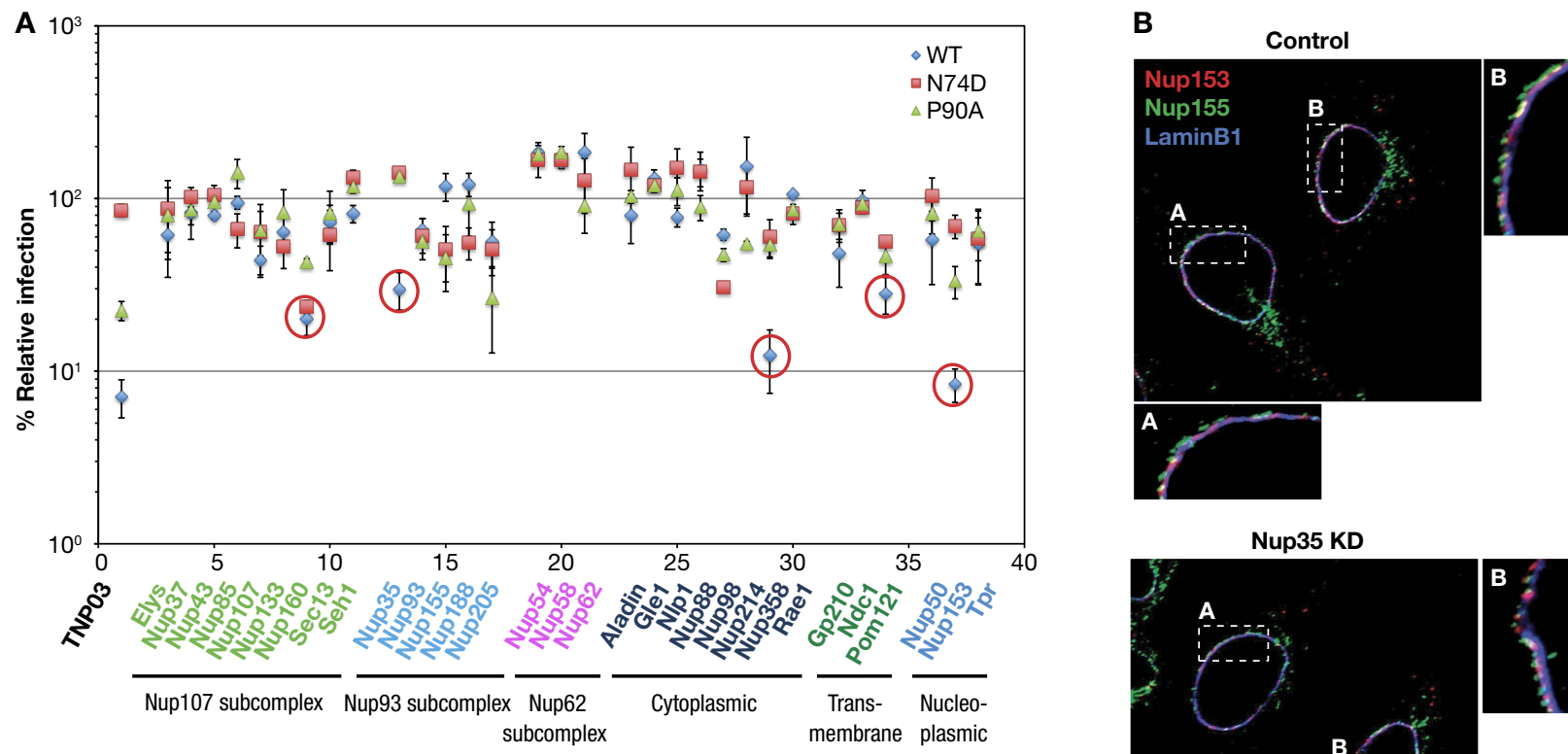

C

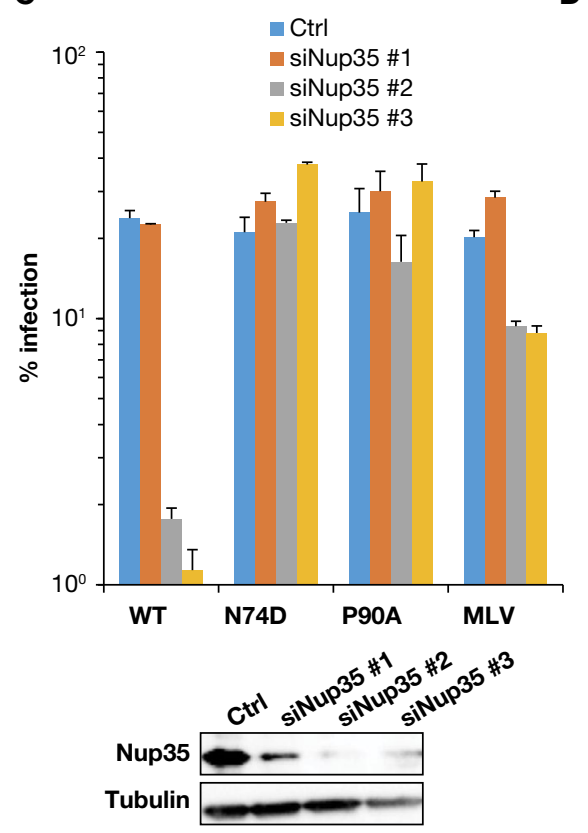

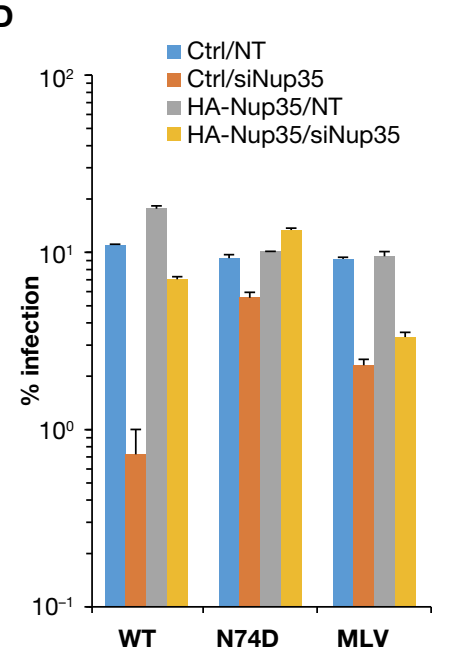

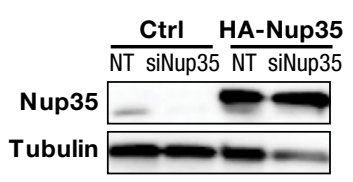

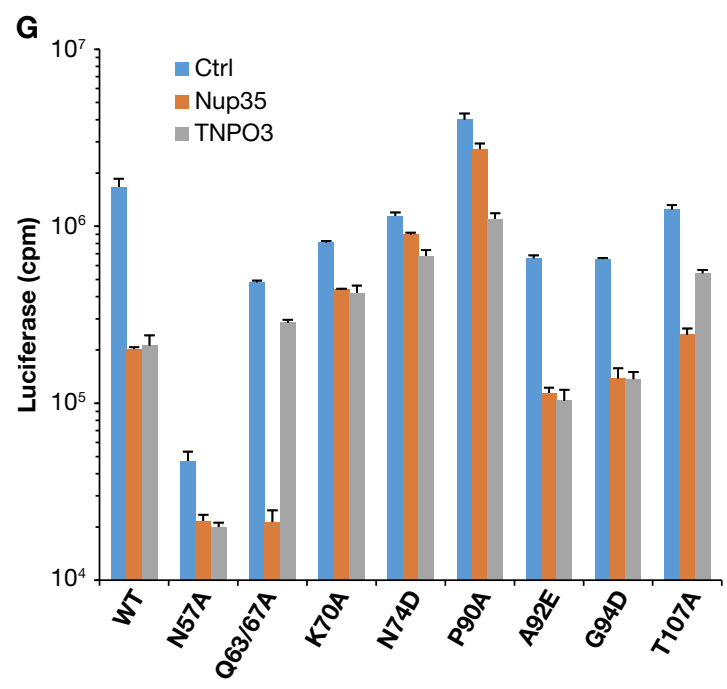
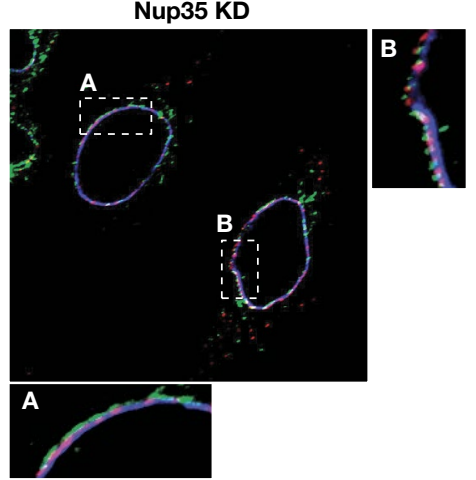
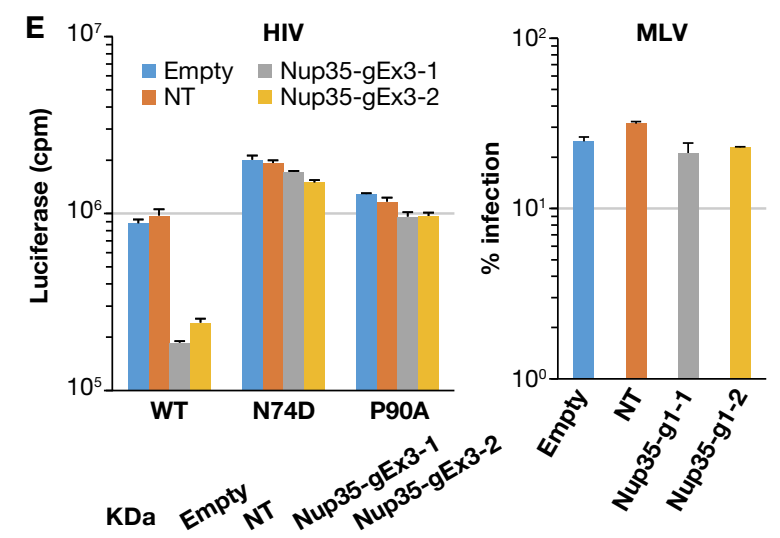
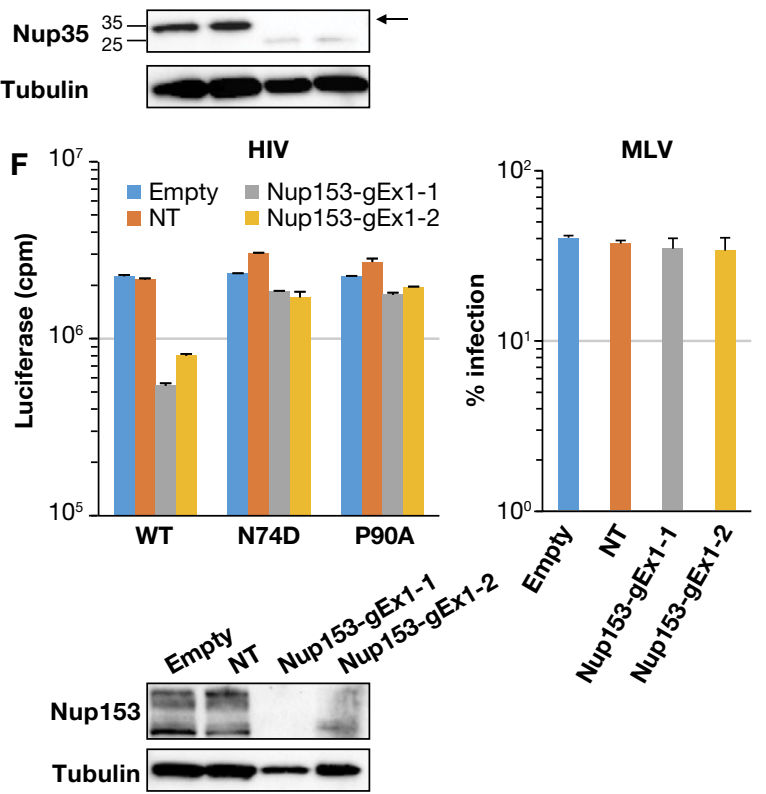
bioRxiv preprint doi: https://doi.org/10.1101/2021.12.02.470925; this version posted December 2, 2021. The copyright holder for this preprint (which was not certified by peer review) is the author/funder, who has granted bioRxiv a license to display the preprint in perpetuity. It is made Figure 2 available under aCC-BY 4.0 International license.
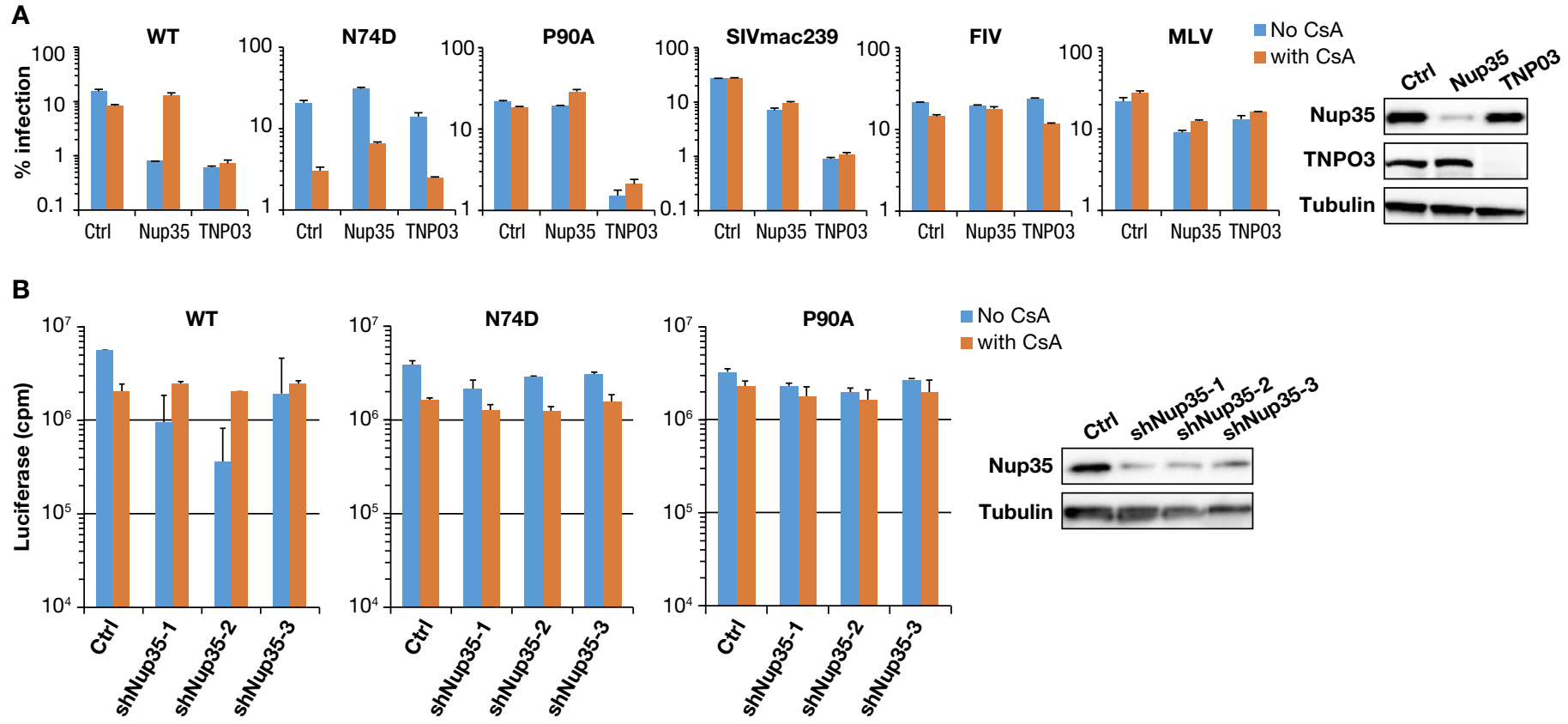

No CsA

with CsA

C
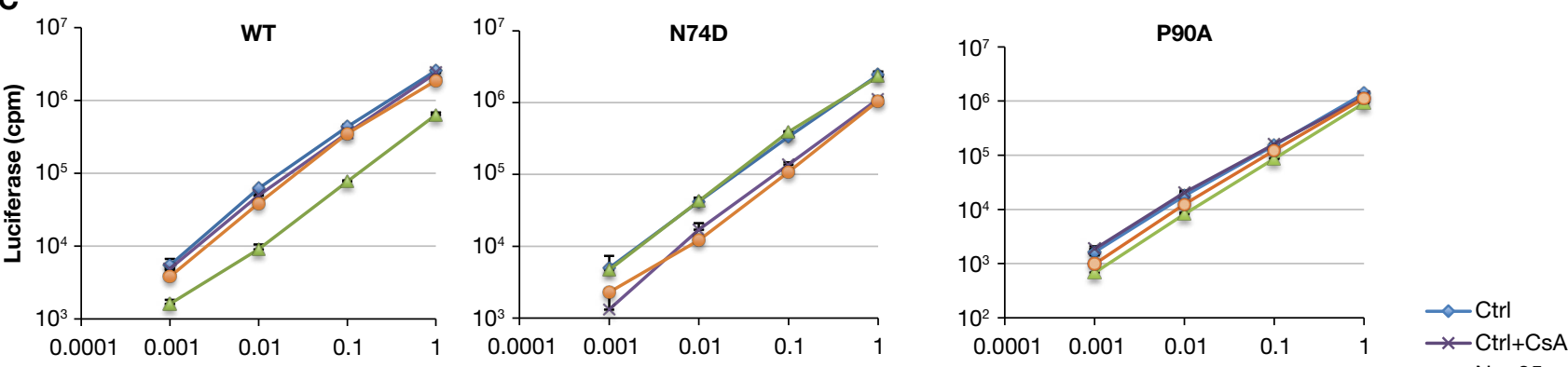

A92E

G94D
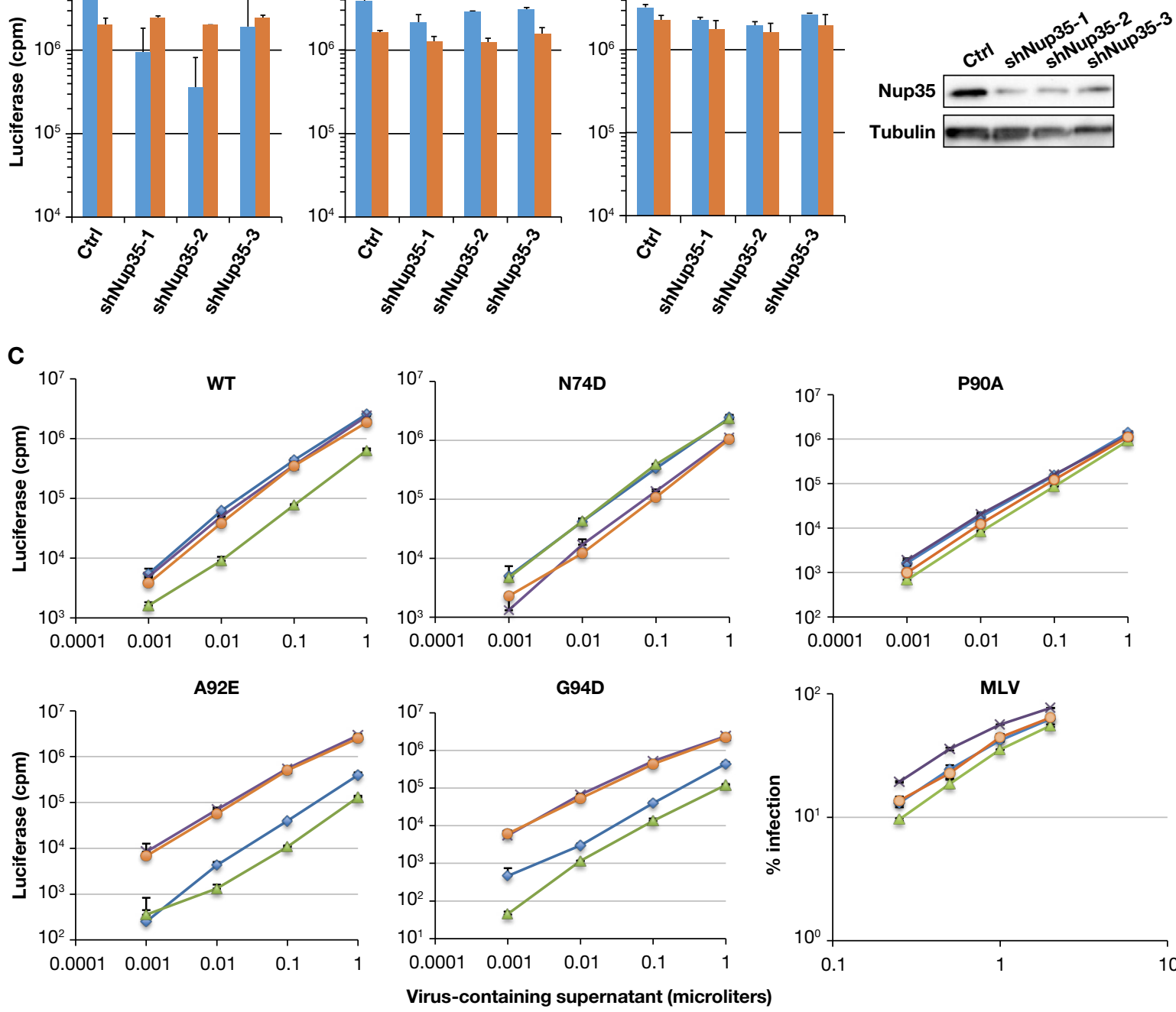

Virus-containing supernatant (microliters)
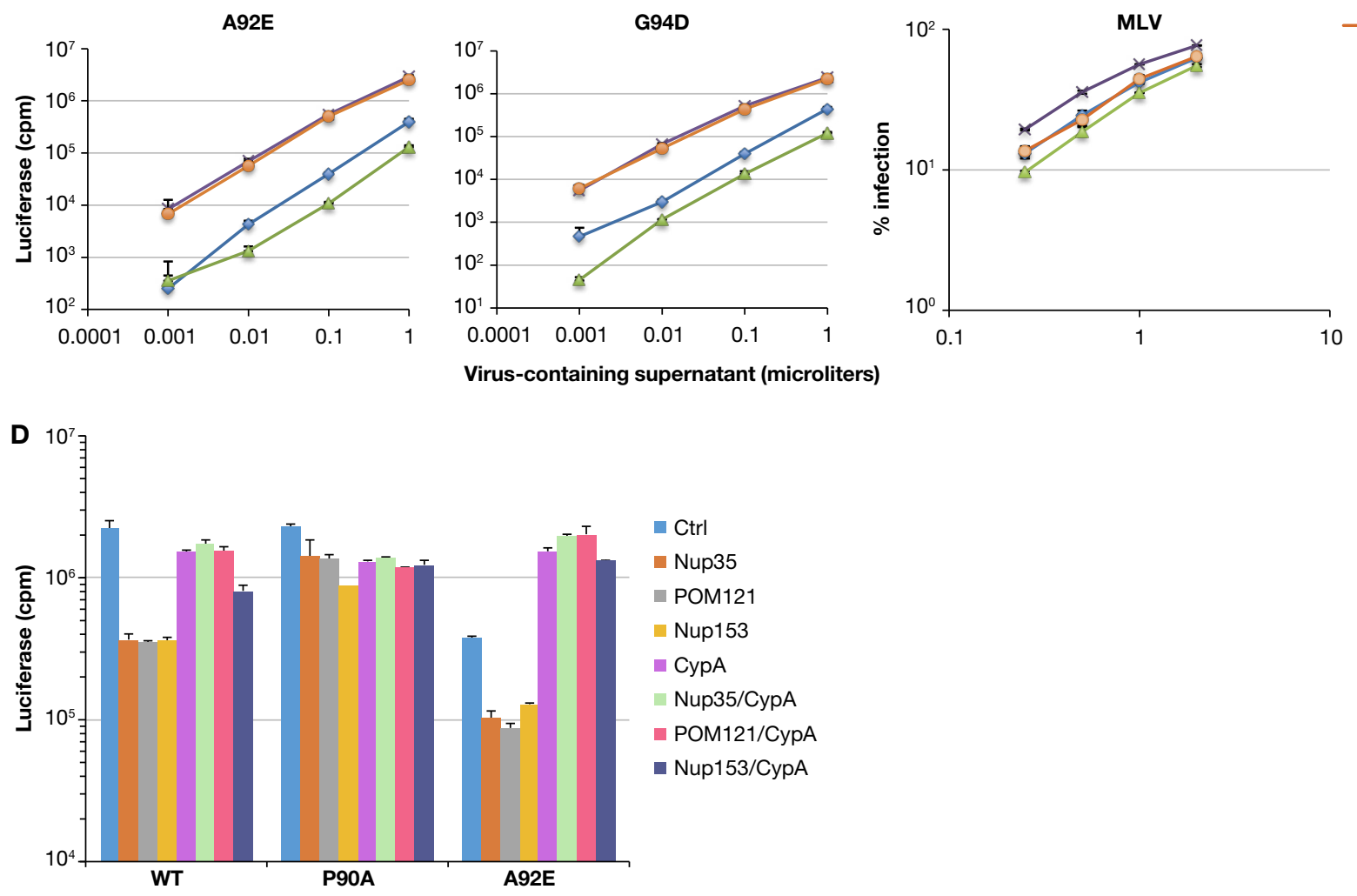
bioRxiv preprint doi: https://doi.org/10.1101/2021.12.02.470925; this version posted December 2, 2021. The copyright holder for this preprint (which was not certified by peer review) is the author/funder, who has granted bioRxiv a license to display the preprint in perpetuity. It is made Figure 3 available under aCC-BY 4.0 International license.
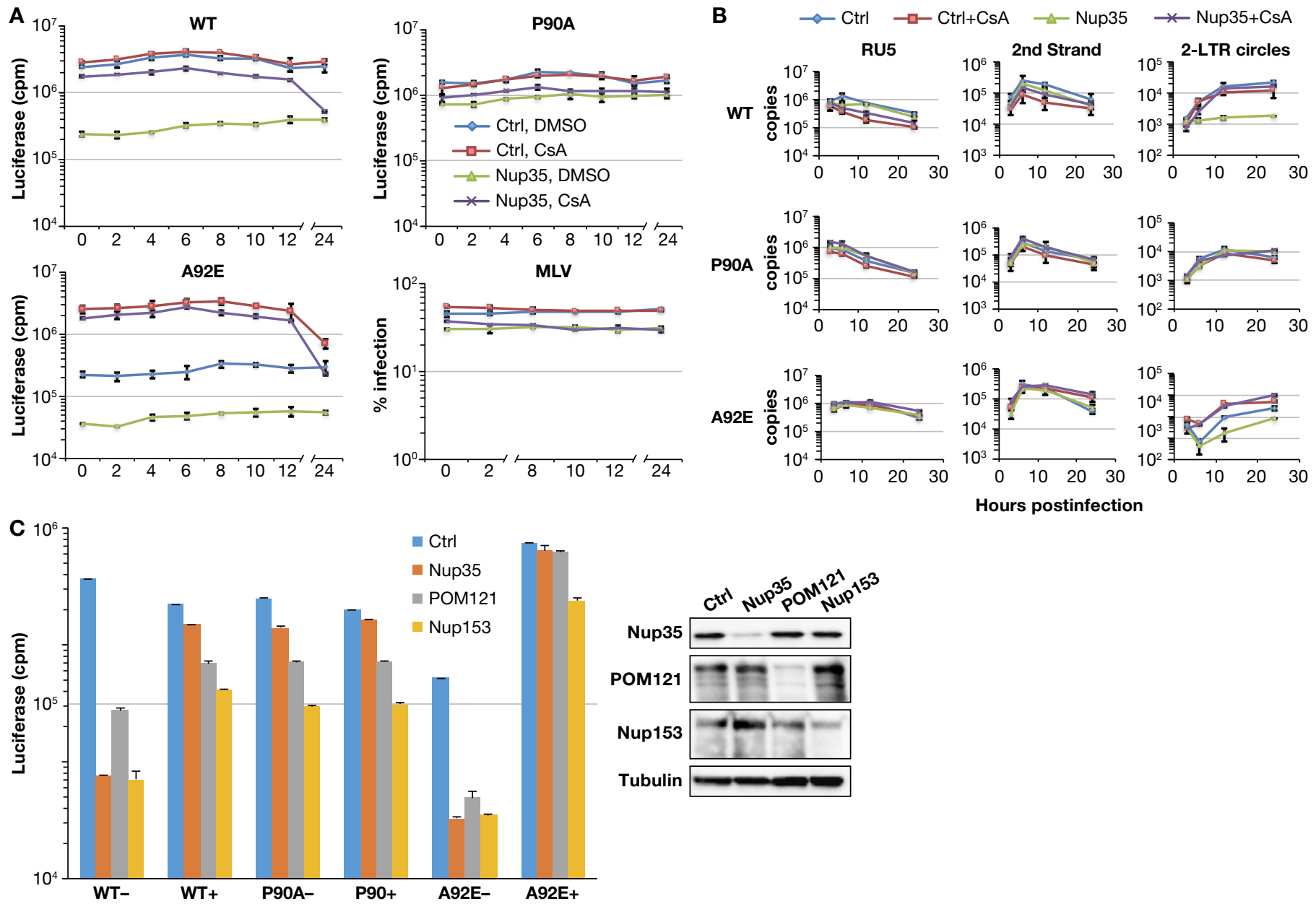

D

CsA (-) ${ }^{\text {Control KD }}$ CsA (+)

E

$\operatorname{CsA}(-)^{N}$
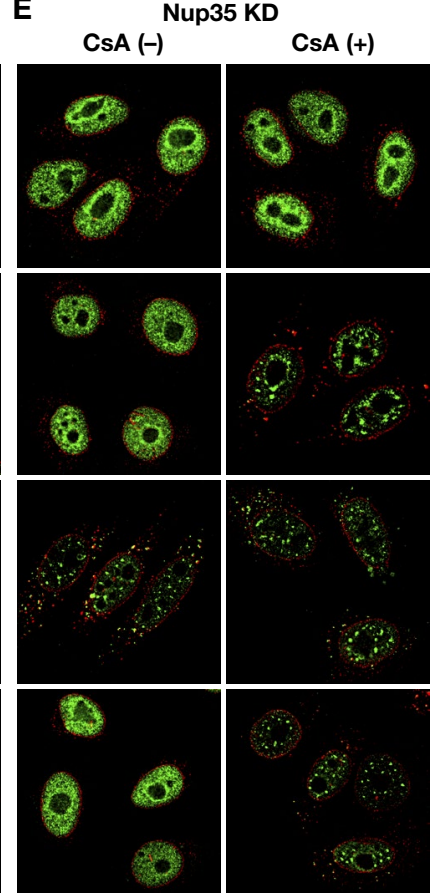

F Pom121 KD
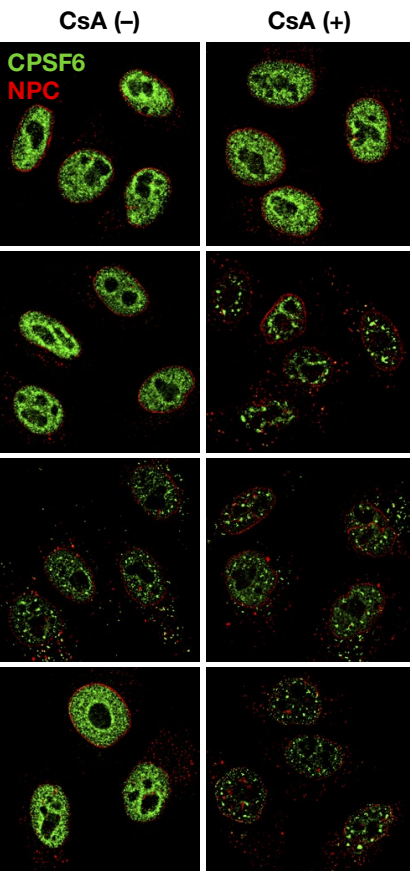

G Nup153 KD
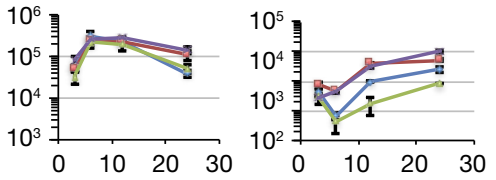

Hours postinfection
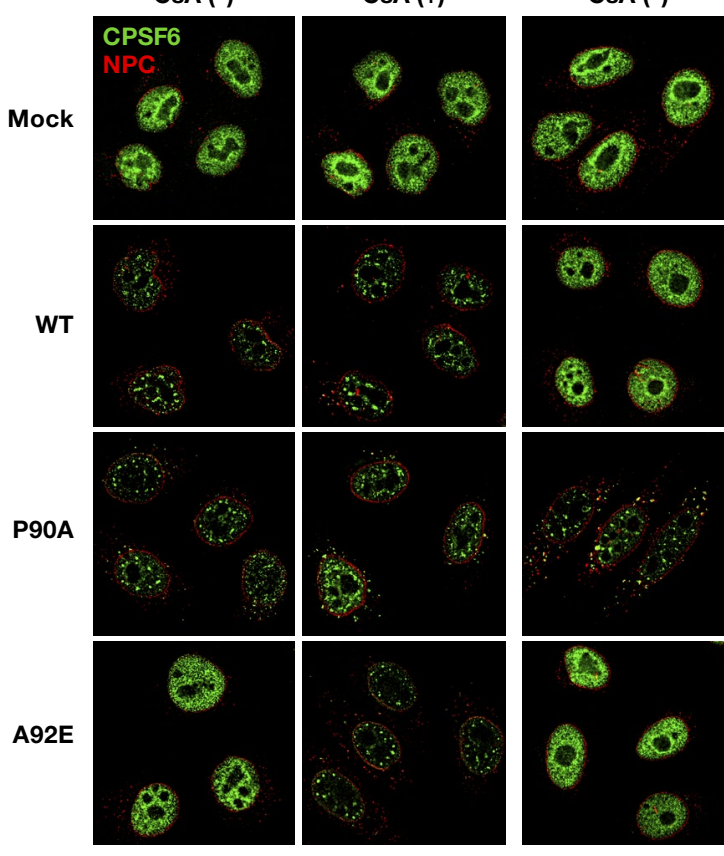
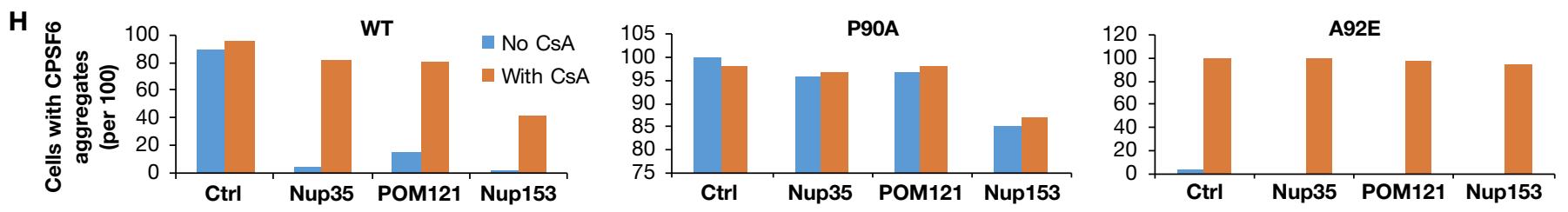
bioRxiv preprint doi: https://doi.org/10.1101/2021.12.02.470925; this version posted December 2, 2021. The copyright holder for this preprint (which was not certified by peer review) is the author/funder, who has granted bioRxiv a license to display the preprint in perpetuity. It is made Figure 4 available under aCC-BY 4.0 International license.
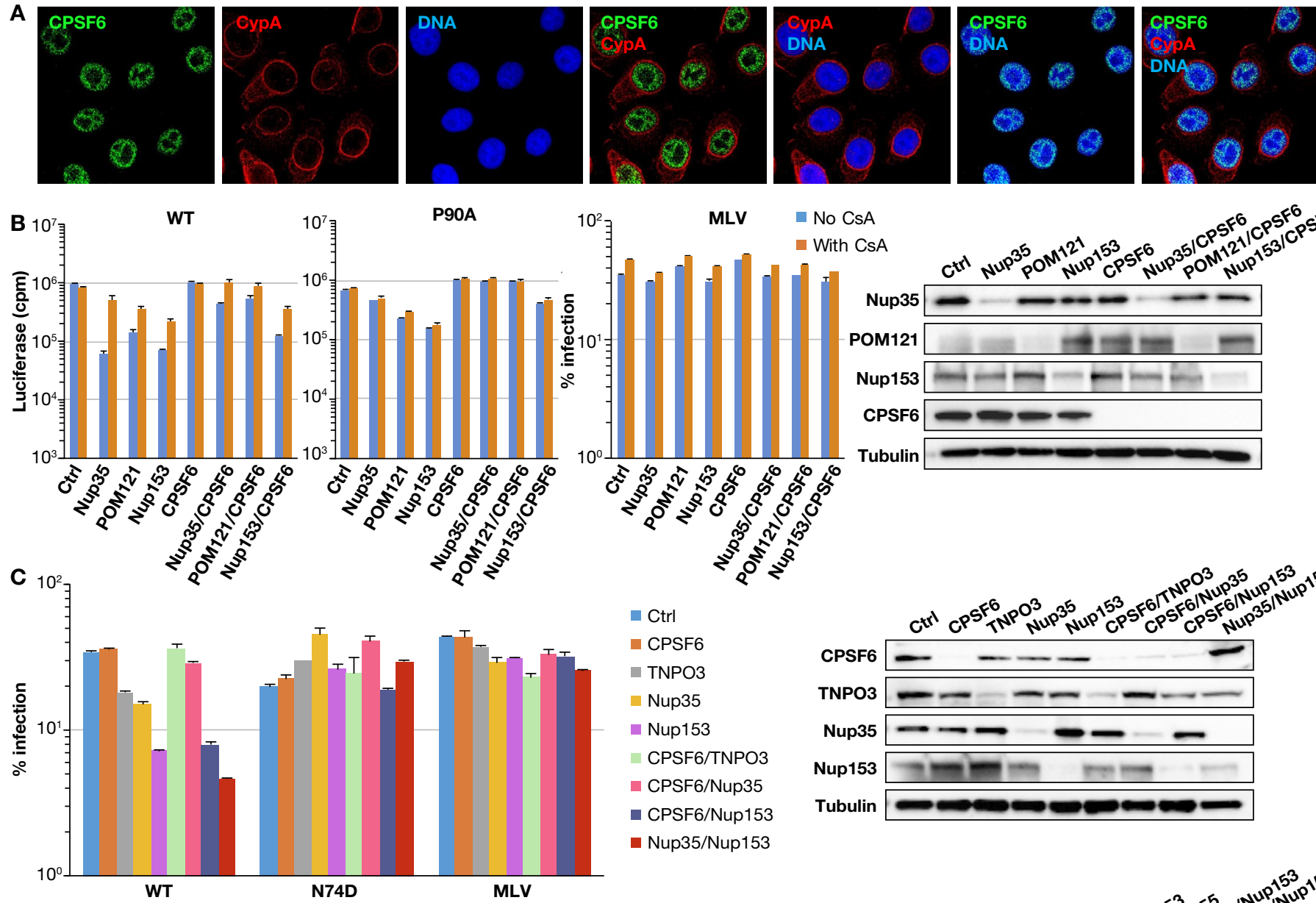

With CsA

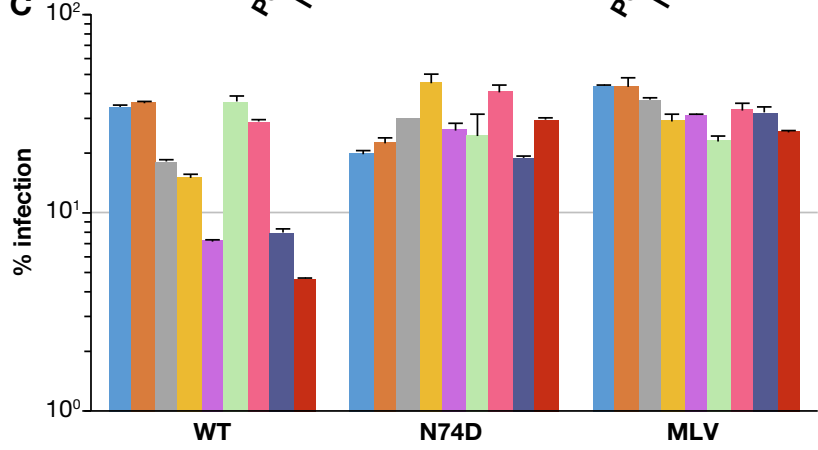

MLV
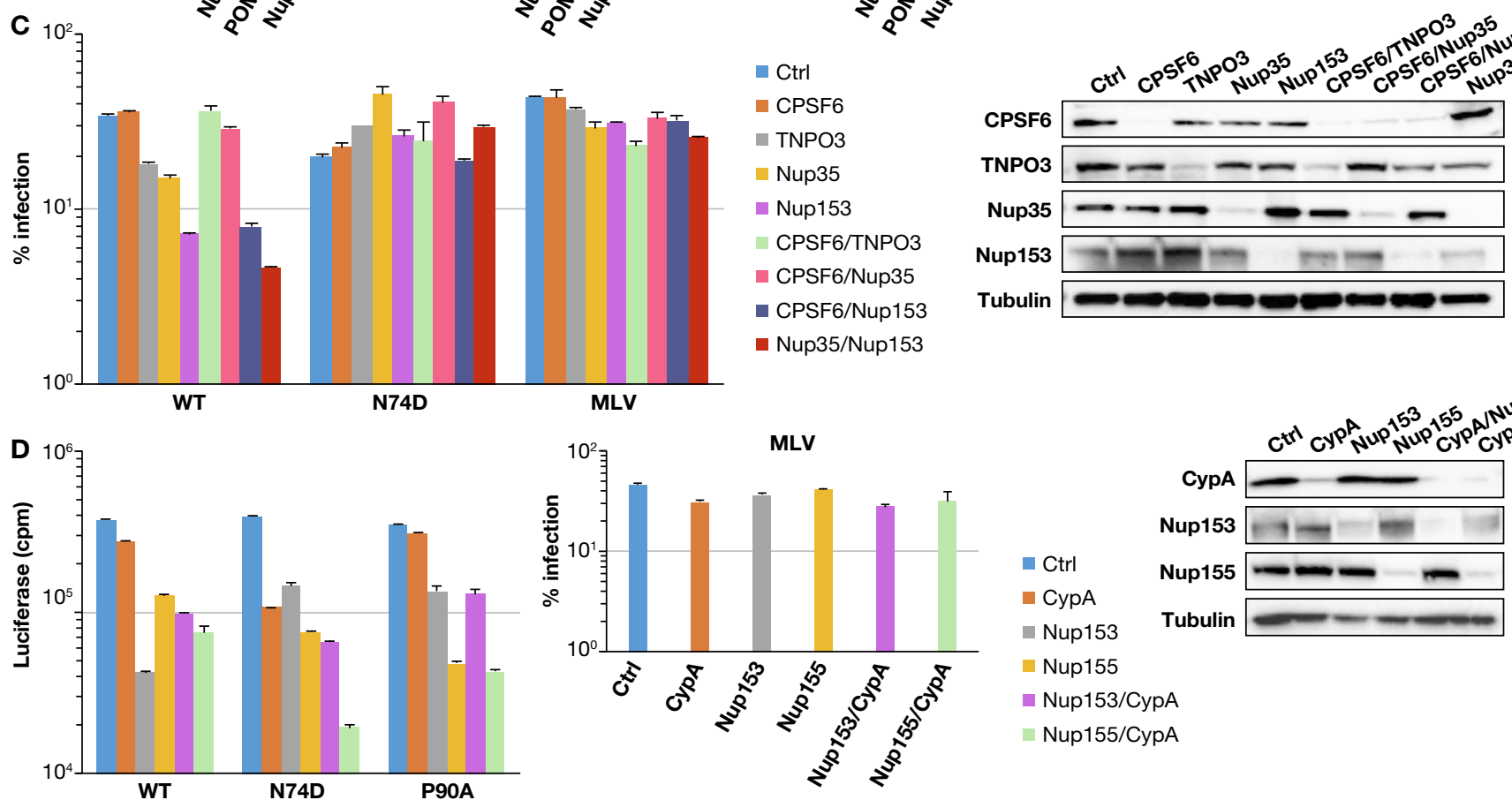

$\mathbf{E}$
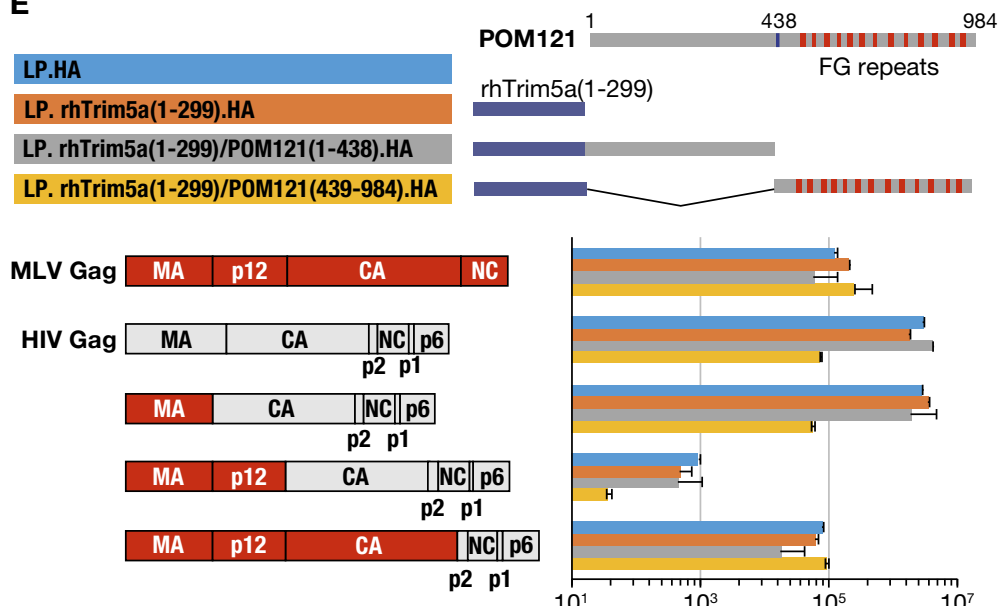

\section{(1)}

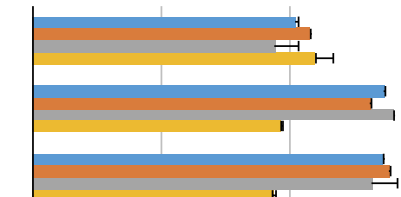

Ctrl

- CypA

- Nup153

- Nup155

Nup153/CypA

Nup155/CypA

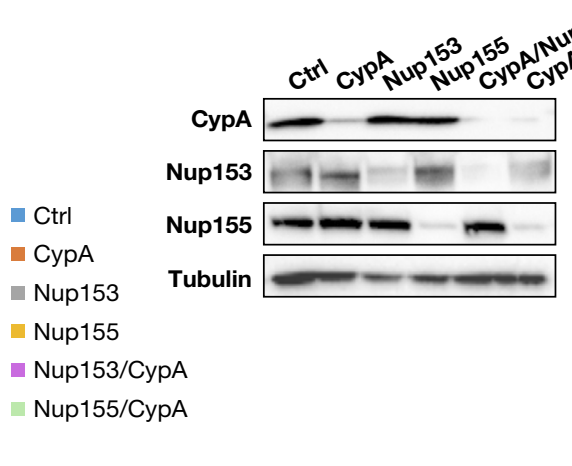

